\title{
The Trimeric Periplasmic Chaperone Skp of Escherichia coli Forms 1:1 Complexes with Outer Membrane Proteins via Hydrophobic and Electrostatic Interactions
}

\author{
Jian $\mathbf{Q u}^{1}$, Christoph Mayer ${ }^{1}$, Susanne Behrens ${ }^{2}$, Otto Holst ${ }^{3}$ \\ and Jörg H. Kleinschmidt ${ }^{1 *}$
}

\author{
${ }^{1}$ Fachbereich Biologie, Universität \\ Konstanz, Universitätsstraße 10 \\ D-78464 Konstanz, Germany \\ ${ }^{2}$ Abteilung Molekulare \\ Genetik und Präparative \\ Molekularbiologie, Institut für \\ Mikrobiologie und Genetik, Georg \\ August Universität Göttingen \\ Grisebachstraße 8, D-37077 \\ Göttingen, Germany \\ ${ }^{3} L G$ Strukturbiochemie \\ Forschungszentrum Borstel \\ Leibniz Zentrum für Medizin \\ und Biowissenschaften, Parkallee \\ 4a, D-23845 Borstel, Germany
}

Edited by I. B. Holland
The interactions of outer membrane proteins (OMPs) with the periplasmic chaperone Skp from Escherichia coli are not well understood. We have examined the binding of Skp to various OMPs of different origin, size, and function. These were OmpA, OmpG, and YaeT (Omp85) from Escherichia coli, the translocator domain of the autotransporter NalP from Neisseria meningitides, FomA from Fusobacterium nucleatum, and the voltagedependent anion-selective channel, human isoform 1 (hVDAC1) from mitochondria. Binding of Skp was observed for bacterial OMPs, but neither for hVDAC1 nor for soluble bovine serum albumin. The Skp trimer formed 1:1 complexes, OMP.Skp 3 , with bacterial OMPs, independent of their size or origin. The dissociation constants of these OMP.Skp 3 complexes were all in the nanomolar range, indicating that they are stable. Complexes of $\mathrm{Skp}_{3}$ with YaeT displayed the smallest dissociation constants, complexes with NalP the largest. OMP binding to $\mathrm{Skp}_{3}$ was $\mathrm{pH}$-dependent and not observed when either Skp or OMPs were neutralized at very basic or very acidic $\mathrm{pH}$. When the ionic strength was increased, the free energies of binding of Skp to OmpA or OmpG were reduced. Electrostatic interactions were therefore necessary for formation and stability of OMP.Skp 3 complexes. Light-scattering and circular dichroism experiments demonstrated that $\mathrm{Skp}_{3}$ remained a stable trimer from $\mathrm{pH} 3$ to $\mathrm{pH} 11$. In the OmpA $\cdot \mathrm{Skp}_{3}$ complex, Skp efficiently shielded tryptophan residues of the transmembrane strands of $\mathrm{OmpA}$ against fluorescence quenching by aqueous acrylamide. Lipopolysaccharide (LPS), a major component of the outer membrane of Gram-negative bacteria, bound to OmpA $\mathrm{Skp}_{3}$ complexes at low stoichiometries. Acrylamide quenching of fluorescence indicated that in this ternary complex, the tryptophan residues of the transmembrane domain of OmpA were located closer to the surface than in binary OmpA $\cdot \mathrm{Skp}_{3}$ complexes. This may explain previous observations that folding of Skp-bound OmpA into lipid bilayers is facilitated in presence of LPS.

Keywords: Skp; outer membrane protein; membrane protein folding; molecular chaperone; protein-protein interactions

*Corresponding author. E-mail address: joerg.helmut.kleinschmidt@uni-konstanz.de.

Abbreviations used: BSA, bovine serum albumin; FomA, major outer membrane protein of Fusobacterium nucleatum; hVDAC1, voltage-dependent anion-selective channel human isoform-1; LPS, lipopolysaccharide; NalP, translocator domain of the autotransporter NalP from Neisseria meningitides; OM, outer membrane; OMP, outer membrane protein; OmpA, outer membrane protein A of E. coli; OmpG, outer membrane protein G of E. coli; PPIases, peptidyl-proly cis/trans isomerases; SurA, the survival factor A; TMD-OmpA, transmembrane domain of OmpA, i.e. amino acid residues 0 176; WT-OmpA, wild type OmpA; YaeT (Omp85), 89 kDa outer membrane protein of E. coli. 


\section{Introduction}

Outer membrane proteins (OMPs) of Gramnegative bacteria are synthesized in the cytosol and translocated in an unfolded form by the SecYEG translocon across the cytoplasmic membrane into the periplasm, before they insert into the outer membrane $(\mathrm{OM})$. In recent years, several studies have demonstrated that the deletion of certain genes encoding periplasmic proteins in Gram-negative bacteria, e.g. deletion of the genes encoding the $17 \mathrm{kDa}$ protein (Skp) or the survival factor A (SurA), results in reduced concentrations of OMPs in the $\mathrm{OM}$, indicating that these periplasmic proteins serve as molecular chaperones in the assembly pathway of OMPs. ${ }^{1}$ In Escherichia coli, expression of these chaperones is under control of either the $\sigma^{\mathrm{E} 2,3}$ or the two-component CpxA/CpxR stress-response system, ${ }^{4,5}$ and upregulated when misfolded OMPs accumulate in the periplasm. Periplasmic chaperones are key factors to prevent aggregation and misfolding of bacterial OMPs. However, little is known about their interaction with integral membrane proteins in solution.

Studies with peptide libraries indicated that periplasmic SurA binds preferentially to peptides containing the amino acid sequence motif aromaticrandom-aromatic with micromolar affinities. Such motifs are found frequently in OMPs. ${ }^{6,7}$ A binding specificity of $\mathrm{Skp}$ has not been reported. When periplasmic cell extracts were run over an affinity column containing unfolded OmpF that was covalently linked to Sepharose, Skp was the major protein that bound to the column. ${ }^{8}$ Upon deletion of the $s k p$ gene, reduced concentrations of OMPs, like outer membrane protein A (OmpA), OmpF, OmpC, or LamB were found in the OM of E. coli. ${ }^{8}$ Skp binds OmpA early after secretion through the cytoplasmic membrane. ${ }^{9}$ It also improves the functional expression of soluble antibody fragments in the periplasm of E. coli. ${ }^{10}$ Periplasmic chaperones do not require ATP, which is not found in the periplasm. ${ }^{11}$

We previously demonstrated that the simultaneous presence of Skp and lipopolysaccharide (LPS) improves insertion and folding of OmpA into phospholipid membranes. ${ }^{12}$ OmpA has been used extensively as a model to study membrane insertion and folding of $\beta$-barrel membrane proteins. Unfolded OmpA in $8 \mathrm{M}$ urea inserts and folds spontaneously into preformed lipid bilayers upon dilution of the urea. ${ }^{13}$ In the presence of $S \mathrm{kp}$, the kinetics of this folding reaction is retarded and folding yields are reduced. ${ }^{12}$ A similar effect is observed, when OmpA folding experiments are performed in the presence of LPS, which is a major component of the OM. However, when Skp and LPS are present simultaneously at low stoichiometries (three Skp and two to seven LPS per OmpA), the folding rates of OmpA are faster than in the absence of both. The concurrent presence of Skp and LPS also leads to larger yields of folded OmpA in lipid bilayers. ${ }^{12}$ The biosyntheses of Skp and LPS are apparently correlated, since the gene fir $A$, which codes for an acyltransferase involved in LPS biosynthesis $^{14,15}$ is located only four bases downstream from the skp gene, mapped at the 4 min region on the chromosome. ${ }^{10}$ Pulse labeling and biochemical reconstitution experiments suggested that LPS may be required for efficient assembly of OMPs such as trimeric PhoE, ${ }^{16}$ and monomeric OmpA, ${ }^{17}$ into outer membranes.

Skp consists of 141 amino acid residues and forms a stable homotrimer, ${ }^{18}$ consistent with the previously determined stoichiometry of Skp in complex with unfolded OmpA. ${ }^{12}$ The trimer resembles a jellyfish and is formed of a tentacle domain with $\alpha$-helical tentacles that protrude about $60 \AA$ from a $\beta$-barrel body termed the association domain. The tentacle domain defines a central cavity, which is accessible from the tip region of the tentacle domain and from the side, where the tentacles are separated by about $25 \AA{ }^{19,20}$ The structural motif of a predicted LPS binding $\operatorname{site}^{20}$ is similar to the one identified in the OMP FhuA of E. coli ${ }^{21}$ and is located in the middle of the Skp tentacles. This predicted binding site is composed of three basic residues (K77, R87, and R88) on the Skp surface.

Here, we have examined Skp binding to several OMPs, namely OmpA, OmpG, and YaeT (also called Omp85) from E. coli, the translocator domain of the autotransporter NalP from Neisseria meningitides, FomA from Fusobacterium nucleatum, and hVDAC1, isoform 1 (Homo sapiens) from mitochondrial OMs. OmpA (35 kDa) is composed of a 171 residue 8 stranded $\beta$-barrel transmembrane domain and a 154 residue periplasmic domain. The $\beta$-barrel of the 301 residue OmpG (33 $\mathrm{kDa})$ consists of $14 \beta$-strands with a central pore, which is capable of transporting large solutes. 22,23 The translocator domain of NalP $(32 \mathrm{kDa})$ forms a 12-stranded TM $\beta$-barrel that contains an N-terminal $\alpha$-helix. ${ }^{24}$ The structure of the $89 \mathrm{kDa}$ YaeT (also called Omp85) is not known, but YaeT consists of a C-terminal TM domain (predicted to contain $12 \mathrm{TM} \beta$-strands ${ }^{25}$ ) and a large 500 residue periplasmic domain. The structure of the YaeT homolog FhaC of Bordetella pertussis was published recently. FhaC forms a 16-stranded $\beta$-barrel. ${ }^{26}$ YaeT (Omp85) is a highly conserved protein that is essential for cell viability and for outer membrane biogenesis. ${ }^{25,27,28}$ FomA (40 kDa), a voltage-dependent general diffusion porin, is predicted to form a 14-stranded TM $\beta$-barrel. ${ }^{29,30}$ hVDAC1 (31 kDa) is the human isoform 1 of the important voltage-dependent anion-selective channel of the mitochondrial outer membrane. ${ }^{31}$ We investigated whether the interaction of Skp of E. coli is specific for OMPs of E. coli or whether Skp recognizes the amino acid sequence of $\beta$-strands independent of the origin of the OMP. For several OMPs, we estimated Skp binding stoichiometries and the free energies of their binding to $S \mathrm{kp}$ to determine whether these are affected by the size of the OMP transmembrane domain. We asked whether binding is exclusively caused by hydrophobic interactions or whether it is also mediated in 
part by electrostatic interactions. Finally, we examined binding of LPS to complexes of Skp and OmpA.

\section{Results}

\section{Fluorescence spectroscopy indicates that Skp interacts with bacterial outer membrane proteins}

Six OMPs of different origin, size and function, OmpA, OmpG, YaeT, NalP, FomA, and hVDAC1, were over-expressed and isolated from $E$. coli in their unfolded forms in $8 \mathrm{M}$ urea. We used fluorescence spectroscopy to investigate their interactions with Skp in aqueous solution after dilution of the urea. For comparison, the interactions of Skp with a soluble protein, bovine serum albumin (BSA), were also examined. Intrinsic Trp fluorescence spectroscopy is an elegant tool to study complex formation of membrane proteins, since the fluorescence emission depends on the hydrophobicity of the microenvironment of the fluorophore. Most OMPs contain tryptophan residues in their hydrophobic transmembrane domains. Skp is only very weakly fluorescent when excited at $290 \mathrm{~nm}$ or above, since it does not contain tryptophan and has only a single tyrosine residue.

The unfolded forms of the OMPs and of BSA in $8 \mathrm{M}$ urea showed fluorescence spectra with an emission maximum at $\lambda_{U} \approx 347 \mathrm{~nm}$ (Figure 1; Table $1)$. Upon strong dilution of the urea, fluorescence maxima of the aqueous forms, $\lambda_{\mathrm{AQ}}$, were shifted
Table 1. Effect of Skp on the fluorescence emission spectra of OMPs at $\mathrm{pH} 8$ (Figure 1)

\begin{tabular}{lccccrr}
\hline OMP & $\mathrm{pI}^{\mathrm{a}}$ & $F_{\mathrm{C}} / F_{\mathrm{AQ}}{ }^{\mathrm{b}}$ & $\begin{array}{c}\lambda_{\mathrm{U}} \\
(\mathrm{nm})^{\mathrm{c}}\end{array}$ & $\begin{array}{c}\lambda_{\mathrm{AQ}} \\
(\mathrm{nm})^{\mathrm{c}}\end{array}$ & $\begin{array}{c}\lambda_{\mathrm{C}} \\
(\mathrm{nm})^{\mathrm{c}}\end{array}$ & $\begin{array}{r}\Delta \lambda \\
(\mathrm{nm})^{\mathrm{d}}\end{array}$ \\
\hline WT OmpA & 5.6 & 2.26 & 347.2 & 344.4 & 338.5 & 5.9 \\
TMD OmpA & 5.5 & 2.20 & 347.3 & 344.3 & 338.6 & 5.7 \\
OmpG & 4.4 & 1.84 & 347.7 & 343.4 & 339.5 & 3.9 \\
YaeT & 4.9 & 1.29 & 347.4 & 338.9 & 338.1 & 0.8 \\
NalP & 8.0 & 1.86 & 348.0 & 344.5 & 339.6 & 4.9 \\
FomA & 8.6 & 1.34 & 347.4 & 339.8 & 339.0 & 0.8 \\
hVDAC1 & 8.6 & 1.03 & 347.2 & 334.9 & 335.3 & -0.4 \\
BSA & 5.6 & 1.03 & 345.4 & 331.1 & 331.5 & -0.4 \\
\hline
\end{tabular}

a Calculated isoelectric point. The pI was calculated using Protparam at the Swissprot database, based on published algorithms. $^{49}$

$\mathrm{b}$ Ratio of the OMP fluorescence intensities at $330 \mathrm{~nm}$ in the presence and in the absence of Skp.

c Wavelength of the fluorescence emission maxima of the unfolded OMP in $8 \mathrm{M}$ urea $\left(\lambda_{U}\right)$, after 100 fold dilution of urea with aqueous buffer $\left(\lambda_{\mathrm{AQ}}\right)$, and of OMP.Skp complexes $\left(\lambda_{\mathrm{C}}\right)$. The maxima were calculated by fitting a lognormal distribution to the experimental spectra. ${ }^{45}$

d $\Delta \lambda=\lambda_{\mathrm{AQ}}-\lambda_{\mathrm{C}}$.

towards shorter wavelengths, ranging from $\lambda_{\mathrm{AQ}} \approx$ 344-340 nm for the bacterial OMPs, to $\lambda_{\mathrm{AQ}} \approx 335 \mathrm{~nm}$ for $\mathrm{hVDAC} 1$ and to $\lambda_{\mathrm{AO}} \approx 331 \mathrm{~nm}$ for BSA, indicating a more hydrophobic environment, caused either by hydrophobic collapse or by formation of folded structure (in the soluble BSA). Fluorescence intensities were higher for YaeT, FomA and BSA, but lower for WT-OmpA, TMD-OmpA, OmpG, NalP, and hVDAC1.

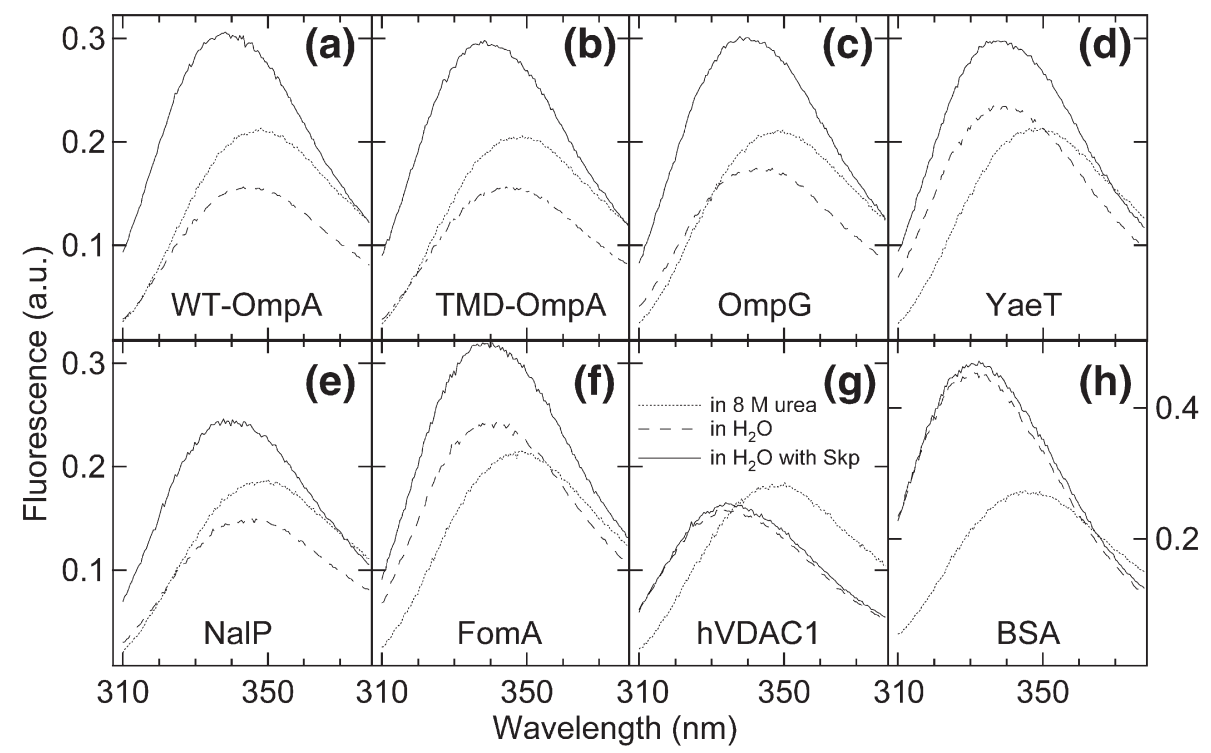

Figure 1. Skp of E. coli interacts with outer membrane proteins of different origin. The fluorescence spectra of: (a) $0.43 \mu \mathrm{M}$ WT-OmpA; (b) $0.41 \mu \mathrm{M}$ TMD-OmpA; (c) $0.18 \mu \mathrm{M}$ OmpG; and (d) $0.14 \mu \mathrm{M}$ YaeT of E. coli; (e) for $0.47 \mu \mathrm{M}$ NalP of N. meningitides; (f) for $0.20 \mu \mathrm{M}$ FomA of F. nucleatum; (g) $0.65 \mu \mathrm{M}$ human VDAC isoform 1 of the mitochondrial outer membrane; and (h) for $0.60 \mu \mathrm{M}$ soluble bovine serum albumin were recorded for the denatured proteins ( $\cdots)$ and for the aqueous forms after 100-fold dilution of the urea either in the absence (- - -) or in the presence of a fivefold molar excess of Skp ( ). All spectra were normalized, dividing fluorescence by the concentration of the OMP and by the number of Trp residues in the OMP. The samples were in $10 \mathrm{mM}$ Tris $\mathrm{HCl}(\mathrm{pH} 8.0)$. Spectra were recorded at $25^{\circ} \mathrm{C}$ at an excitation wavelength of $295 \mathrm{~nm}$. 
When a fivefold molar excess of Skp was added to the aqueous forms of the OMPs after dilution of the denaturant, the fluorescence intensities of all bacterial OMPs increased compared to that of either their aqueous or urea-unfolded forms, indicating their binding to Skp. There was no change in the fluorescence maxima of hVDAC1 or BSA, suggesting that these two proteins do not bind to Skp. The wavelengths of the fluorescence maxima of the OMP.Skp complexes, $\lambda_{C}$, were even shorter, 338$340 \mathrm{~nm}$ (Figure 1; Table 1), which demonstrated that the environment of the fluorescent tryptophan residues of the OMPs is more hydrophobic in complex with Skp. The strongest increase in fluorescence (2.2-2.3-fold) and the largest shift of the emission maximum, 5.7-5.9 nm, was observed when Skp was reacted with TMD-OmpA or WT-OmpA, respectively (Table 1).

\section{Trimeric Skp forms 1:1 complexes with outer membrane proteins}

Our previous work ${ }^{12}$ described a 3:1 stoichiometry for binding of Skp to aqueous OmpA, which is consistent with the more recent observation that Skp is a trimer in solution. ${ }^{18}$ However, the binding stoichiometry may depend on the size of the OMP or its transmembrane domain. To obtain stoichiometries and to estimate the strength of Skp binding, we recorded fluorescence spectra of the aqueous forms of each OMP after dilution of the urea as a function of the molar Skp/OMP ratio until no further in- crease of the fluorescence intensity was observed (Figure 2). Skp formed complexes with five OMPs: OmpA (WT-OmpA and TMD-OmpA), OmpG, YaeT of E. coli, NalP of N. meningitides, and FomA of F. nucleatum. When OMPs were titrated with Skp, their emission spectra were a superposition of the fluorescence spectra of the Skp-bound and of the free form of the OMP. Both the free and the bound OMP contributed to the fluorescence signal of the sample (Figure 1). Since changes in fluorescence at $330 \mathrm{~nm}\left(F_{330} \mathrm{~nm}\right)$ were relatively large and $F_{330} \mathrm{~nm}$ increased linearly for all OMPs upon initial titration with Skp (Figure 2), we monitored binding at this wavelength. $F_{330} \mathrm{~nm}$ was analyzed as a linear combination of the fluorescence contributions of bound and free OMP as described in Materials and Methods. For analysis, binding functions (equation (2)) were fit to the fluorescence intensities at $330 \mathrm{~nm}$ as a function of the $\mathrm{Skp} / \mathrm{OMP}$ ratio. The fits to these binding data converged for four OMPs (Figure 2), but not for FomA (data not shown). The stoichiometries, association constants, dissociation constants, and free energies of Skp binding that were obtained from the fits are given in Table 2 for each of these OMPs. The stoichiometries were all similar and demonstrated formation of stable 1:3 complexes, OMP.Skp 3 . The size of the OMPs varied from $19 \mathrm{kDa}$ (TMD-OmpA) to $89 \mathrm{kDa}$ (YaeT) and the number of transmembrane $\beta$-strands of the folded OMP ranged from 8 in OmpA to 14 in OmpG. These differences are obviously of no consequence for the binding stoichiometry. Since there was neither a

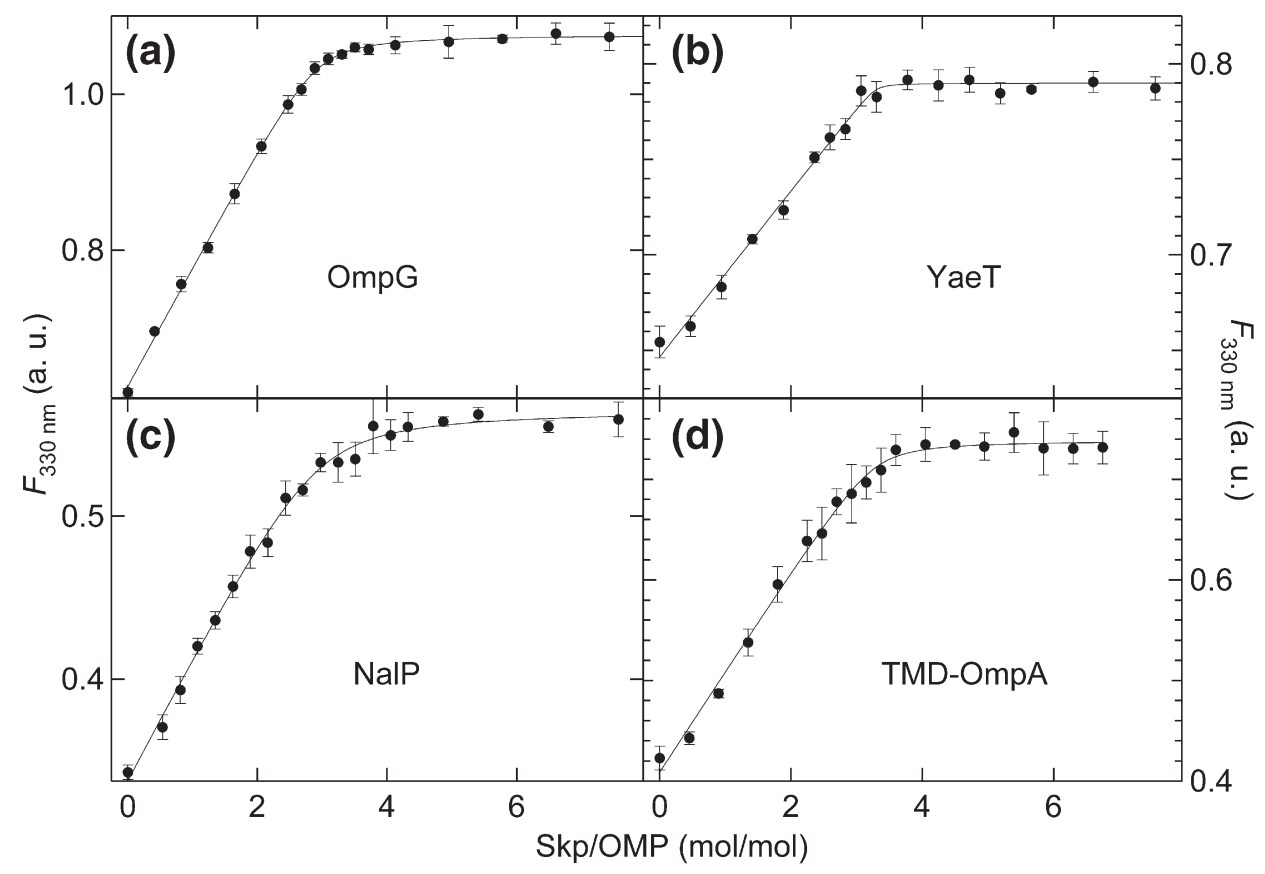

Figure 2. Binding of Skp to the aqueous forms of various outer membrane proteins in solution. The fluorescence signals at $330 \mathrm{~nm}$ of the aqueous forms of (a) OmpG, (b) YaeT, (c) NalP, and (d) TMD-OmpA increased as a function of the molar Skp/OMP ratio until saturation levels were reached. At each Skp/OMP ratio, three separate samples were prepared and fluorescence spectra were recorded to obtain averaged fluorescence intensities. To calculate stoichiometries and binding constants, binding isotherms given by equation (2) were fit to the data ( ). Spectra were recorded at $25{ }^{\circ} \mathrm{C}$ and at an excitation wavelength of $290 \mathrm{~nm}$. 
Table 2. Stoichiometry and free energy of Skp binding to OMPs

\begin{tabular}{|c|c|c|c|c|c|c|}
\hline OMP & $M^{\mathrm{a}}$ & $Z_{\mathrm{TM} \beta \mathrm{S}}{ }^{\mathrm{b}}$ & $n^{\mathrm{c}}$ & $K_{\text {ass }}\left(\mu \mathrm{M}^{1}\right)^{\mathrm{d}}$ & $K_{D}(\mathrm{nM})^{\mathrm{d}}$ & $\Delta G_{\text {ass }}(\mathrm{kJ} / \mathrm{mol})^{\mathrm{e}}$ \\
\hline WT OmpA & 35 & 8 & $2.8 \pm 0.1$ & $46 . \pm 35$ & $22 . \pm 16$ & $-43.0 \pm 2$ \\
\hline WT OmpA (1 M NaCl) & 35 & 8 & $3.1 \pm 0.2$ & $12 . \pm 4$ & $83 . \pm 30$ & $-40.3 \pm 1.0$ \\
\hline TMD OmpA & 19 & 8 & $3.3 \pm 0.1$ & $49 . \pm 35$ & $20 . \pm 15$ & $-43.9 \pm 3.2$ \\
\hline OmpG & 33 & 14 & $2.9 \pm 0.1$ & $82 . \pm 20$ & $12 . \pm 3$ & $-45.2 \pm 0.7$ \\
\hline OmpG (0.1 M NaCl) & 33 & 14 & $2.9 \pm 0.2$ & $19 . \pm 9$ & $53 . \pm 17$ & $-41.6 \pm 1.6$ \\
\hline OmpG (0.5 M NaCl) & 33 & 14 & $2.8 \pm 0.3$ & $12 . \pm 6$ & $83 . \pm 27$ & $-40.3 \pm 1.8$ \\
\hline YaeT $^{\mathrm{f}}$ & 89 & $u^{\mathrm{f}}$ & $3.3 \pm 0.1$ & $2900 . \pm 900$ & $0.3 \pm 0.1$ & $-54.0 \pm 0.9$ \\
\hline NalPg & 32 & 12 & $3.0 \pm 0.1$ & $20 . \pm 8$ & $50 . \pm 20$ & $-42.4 \pm 1.3$ \\
\hline
\end{tabular}

a Molecular mass, $M$ in $\mathrm{kDa}$.

b Number of transmembrane $\beta$ strands, $Z_{\mathrm{TM} \beta \mathrm{S}}$, of the folded form of the OMP.

c Stoichiometry, $n$, estimated from fits to the data shown in Figures 2 and 5.

d Estimated binding constants for association $K_{\mathrm{ass}}$ and dissociation $K_{\mathrm{D}}=1 / K_{\mathrm{ass}}$

e Estimated free energy of binding for the association reaction.

f A structure of the YaeT TM domain has not been published to date and the number of strands in the TM domain is unkown.

g Translocator domain of NalP, residues 7761083.

difference in the stoichiometry or in the free energy of binding for TMD-OmpA (176 residues) and WTOmpA (325 residues), ${ }^{12}$ Skp recognizes the transmembrane domain of OmpA specifically. Dissociation constants were in the nanomolar range, indicating stable complexes. The free energy of complex formation, $\Delta G_{\text {ass }}$, ranged from $-42 \mathrm{~kJ} / \mathrm{mol}$ to $-45 \mathrm{~kJ} / \mathrm{mol}(-10 \mathrm{kcal} / \mathrm{mol}$ to $-11 \mathrm{kcal} / \mathrm{mol})$ for TMD-OmpA, WT-OmpA, NalP and OmpG, but binding was stronger for YaeT with $\Delta G \approx-54 \mathrm{~kJ} / \mathrm{mol}$ $(-13 \mathrm{kcal} / \mathrm{mol})$.

\section{Skp binding to outer membrane proteins is pH-dependent}

OMPs develop their hydrophobic surface upon formation of their amphipathic $\beta$-strands. The antiparallel strands form a $\beta$-barrel, in which the polar residues are oriented towards the interior lumen and the hydrophobic residues are oriented towards the fatty core of the lipid bilayer. The average hydrophobicity of OMPs is low when compared to membrane proteins of the cytoplasmic membrane and closer to the average hydrophobicity of soluble proteins. In complex with Skp, OmpA remained largely unfolded. ${ }^{12}$ It is therefore conceivable that the interactions between Skp and aqueous OMPs are not entirely of a hydrophobic nature. Skp is a very basic protein and positively charged (theoretical pI 2.6-10.5), while OMPs of E. coli have isoelectric points ranging from $\mathrm{pI} 4.5$ to $\mathrm{pI} 6$ and are negatively charged. These opposite net charges suggest electrostatic attraction between Skp and OMPs. Skp has a large cavity inside its tentacle domain that contains hydrophobic patches and could shield the hydrophobic residues of OMPs from the aqueous space while preserving them in their monomeric forms for subsequent membrane insertion. For these reasons, the formation of stable and stoichiometric complexes of unfolded aqueous OMPs with Skp may be driven by electrostatic interactions, or by the hydrophobic effect, or by both.

To investigate whether the charged residues of Skp and OMPs participate in complex formation, we recorded the fluorescence spectra of four aqueous
OMPs in the absence and in the presence of Skp as a function of $\mathrm{pH}$, including the regions above and below the isoelectric points of Skp and OMPs, respectively. The fluorescence intensities at $330 \mathrm{~nm}$, $F_{330 \mathrm{~nm}}$, and the wavelengths, $\lambda_{\text {max }}$, of the emission maxima of OMPs and OMP.Skp 3 complexes were pH-dependent (Figure 3).

Skp did not interact with aqueous hVDAC1 (pI 8.6). In the absence of $S \mathrm{kp}, F_{330 \mathrm{~nm}}$ and $\lambda_{\max }$ of the fluorescence spectra of hVDAC1 changed only slightly between $\mathrm{pH} 4$ and $\mathrm{pH} 9$ (Figure 3(d)). At both ends of the $\mathrm{pH}$ range, in particular at $\mathrm{pH} \geq 10$, fluorescence decreased and $\lambda_{\max }$ increased, indicating a more polar environment and increased water accessibility of the fluorescent tryptophan residues. This is caused by side-chain deprotonation (at $\mathrm{pH}$ $>9$ ) or protonation (at $\mathrm{pH}<5$ ), which both increase the net charge of hVDAC1 (pI 8.6). Independent of $\mathrm{pH}$, the addition of Skp had little or no effect on the fluorescence spectra of hVDAC1.

The fluorescence properties of the aqueous forms of the bacterial OMPs (Figure 3(a)-(c)) displayed a different dependence on $\mathrm{pH}$ in the absence of Skp. From $\mathrm{pH} 6$ to $\mathrm{pH} \mathrm{10,} \mathrm{the} \mathrm{fluorescence} \mathrm{maxima} \mathrm{of}$ these OMPs were located at a longer wavelength than the emission maxima of hVDAC1, indicating at least partial exposure of the tryptophan residues of OmpA, NalP, and FomA to the aqueous solution. When the $\mathrm{pH}$ was lowered to 4 or $5, F_{330} \mathrm{~nm}$ increased sharply, and $\lambda_{\max }$ decreased strongly, for OmpA, NalP, and FomA, indicating that the fluorescent tryptophan residues became more shielded from polar interactions and had a more hydrophobic environment at this $\mathrm{pH}$. In the absence of Skp, WT-OmpA and NalP displayed one major titration (around $\mathrm{pH}$ 5.5) of Trp exposure to the aqueous environment. The $\mathrm{pH}$-dependent changes of the FomA spectra indicated two titrations, one between $\mathrm{pH} 5$ and $\mathrm{pH} 7$, and a second between $\mathrm{pH} 9$ and $\mathrm{pH}$ 11. This suggested that there are two different conformations of FomA in different regions of $\mathrm{pH}$ that display a different surface exposure of tryptophan to the aqueous solution.

Binding of Skp to WT-OmpA, NalP, and FomA was $\mathrm{pH}$-dependent, as indicated by the changes in 


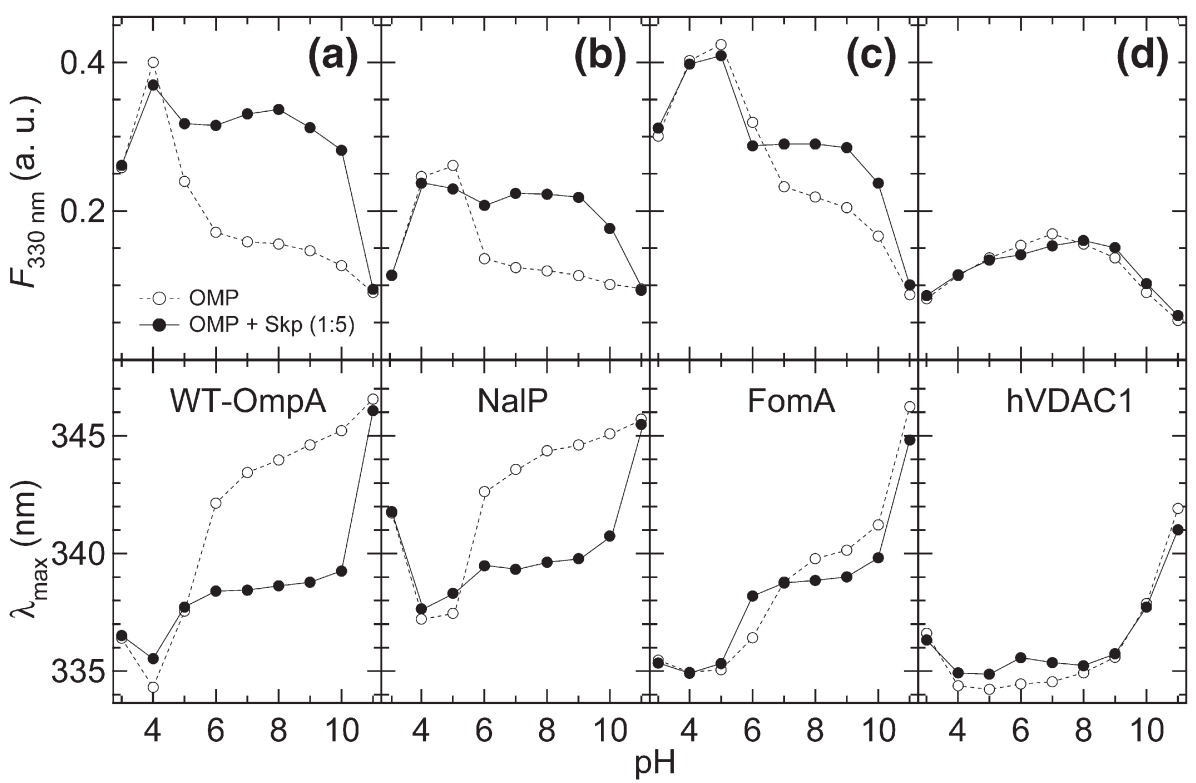

Figure 3. Effect of $\mathrm{pH}$ on the binding of Skp to outer membrane proteins. To examine the $\mathrm{pH}$-dependence of binding of Skp to OMPs (a) WT-OmpA, (b) NalP, (c) FomA and (d) hVDAC1, fluorescence spectra of these OMPs were recorded for the aqueous forms in the absence $(O)$ and in the presence $(0)$ of a fivefold molar excess of Skp as a function of $\mathrm{pH}$ between $\mathrm{pH} 3$ and $\mathrm{pH}$ 11. Urea was diluted 100-fold to a residual concentration of $80 \mathrm{mM}$. The upper panels show the fluorescence intensities at $330 \mathrm{~nm}$ and the lower panels show the wavelengths of the intensity maxima of the spectra upon excitation at $295 \mathrm{~nm}$.

the Trp fluorescence spectra upon addition of Skp (Figure 3(a)-(c)). From pH 6 to pH 10, Skp binding resulted in strongly increased OMP fluorescence and in a shift of $\lambda_{\max }$ towards shorter wavelength, indicating a more hydrophobic environment of the tryptophan residues of these OMPs in the complexes. Binding of Skp to FomA led to smaller fluorescence changes than Skp binding to OmpA or NalP. In the absence of $S k p$, the tryptophan residues of the aqueous form of FomA are apparently less exposed to water than those of OmpA and NalP and, therefore, fluorescence spectra of FomA were less affected by Skp binding.

Above $\mathrm{pH} \sim 10.5$, the positively charged lysine residues of $\mathrm{Skp}$ deprotonate and become neutral. Skp did not bind to OMPs at $\mathrm{pH} 11$ or above, indicating a contribution of charge interactions to the free energy of OMP binding to Skp. Binding of OmpA or NalP to Skp was not detected below pH 5. While this is close to the $\mathrm{pI}$ of OmpA, NalP and FomA have much higher theoretical isoelectric points, namely at pI 8.0 and at pI 8.6, respectively. The charge distribution in the aqueous forms of these OMPs after dilution of the denaturant is unknown and it cannot be excluded that it is asymmetric. The titrations of both OMPs in the absence of Skp indicated conformational transitions below $\mathrm{pH}$ 6 in both NalP and FomA as a consequence of protonation.

\section{Skp is a stable trimer over a wide range of $\mathrm{pH}$ values}

We next performed dynamic light-scattering experiments to exclude the possibility that the ob- served $\mathrm{pH}$-dependence of OMP binding to Skp may be caused by a loss of the trimeric structure of Skp at very acidic or very basic $\mathrm{pH}$. The hydrodynamic radius of $\mathrm{Skp}$ remained unaltered at $R_{\mathrm{H}}=3.5 \mathrm{~nm}$ over the entire $\mathrm{pH}$ range from 3 to 11 (Figure 4). The experimentally obtained molecular mass of the Skp particles $M_{\exp }\left(\mathrm{Skp}_{3}\right)$ was $\approx 54 \mathrm{kDa}$ as calculated using equation (1). This is consistent with the trimeric structure of $\mathrm{Skp}$ (calculated from the amino acid sequence as $\left.M\left(\mathrm{Skp}_{3}\right)=47 \mathrm{kDa}\right)$. Skp contains a central cavity within its tentacle domain and therefore the specific volume of Skp is likely larger than that of a typical protein. An overestimation of $M_{\text {exp }}\left(\mathrm{Skp}_{3}\right)$ is therefore not surprising. The $\mathrm{pH}-$ independence of $R_{\mathrm{H}}$ and of CD spectra of Skp (Figure 4(b)) indicated that the Skp trimer is stable and resists changes of $\mathrm{pH}$.

\section{High ionic strength reduces the stability of $\mathrm{Skp}_{3}$-OMP complexes}

To further examine the electrostatic contribution to the binding of $\mathrm{Skp}_{3}$ to OMPs, we titrated OmpA (Figure 5(a)) and OmpG (Figure 5(b)) with Skp at different concentrations of salt. The fluorescence intensities of OmpA and OmpG increased at higher concentrations of $\mathrm{NaCl}$, but $\lambda_{\mathrm{AQ}}$ decreased only slightly to about $340 \mathrm{~nm}$ (data not shown), indicating that the tryptophan residues are still partially exposed to the aqueous environment at high concentrations of salt. Skp binds to these OMPs even at $1 \mathrm{M} \mathrm{NaCl}$. At high ionic strength, a more gradual saturation of the binding functions was observed with OmpA (Figure 5(a), $\square$ ) and with OmpG (Figure 5(b), $\triangle, \diamond)$. At a low concentration of 

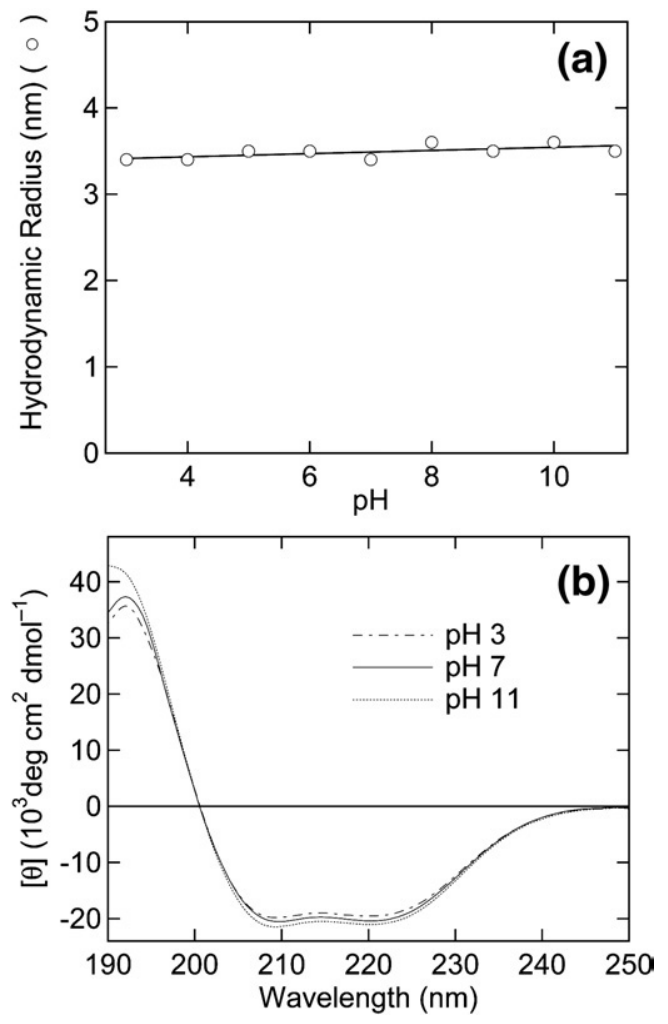

Figure 4. Skp forms a stable trimer from $\mathrm{pH} 3$ to $\mathrm{pH}$ 11. (a) Light-scattering experiments demonstrate that the hydrodynamic radius of Skp shows no variation between $\mathrm{pH} 3$ and $\mathrm{pH}$ 11. Experiments were performed at $25^{\circ} \mathrm{C}$ at an Skp concentration of $0.13 \mathrm{mM}$. (b) CD spectra of Skp at $\mathrm{pH} 3(-\cdots)$, pH $7($ ), and $\mathrm{pH} 11(\cdots)$, and similar CDspectra at $\mathrm{pH} 4,5,6,8,9$, and 10 (data not shown), indicate that the secondary structure of Skp does not change over the entire $\mathrm{pH}$ range.

salt, the binding data leveled off sharply (Figure 5(a) and (b), $\bigcirc$ ). The more gradual leveling at high ionic strength already suggested weaker interactions between the binding partners. As expected, fits of the binding functions to the data resulted in lower association constants and the free energies of binding (association) were reduced by $\sim 3 \mathrm{~kJ} / \mathrm{mol}$ for OmpA and by $\sim 5 \mathrm{~kJ} / \mathrm{mol}$ for OmpG (Table 2). Although the difference in the free energies was small, the association constant for OmpG binding was reduced two- to fourfold. The actual contribution of electrostatic interactions to the binding of Skp to OMPs was likely much larger, since the presence of salt does not completely shield the charged residues of the proteins. In comparison, the protonation of the anionic residues at low $\mathrm{pH}$ or the deprotonation of cationic residues at high $\mathrm{pH}$ completely altered the charge-state of the proteins.

\section{LPS binds to OmpA.Skp ${ }_{3}$ complexes}

LPS facilitated folding of Skp-bound OmpA into lipid bilayers, ${ }^{12}$ and the crystal structure of $E$. coli Skp ${ }^{19,20}$ indicated a possible LPS-binding site in the tentacle domain of each Skp monomer, which is conserved in Skp orthologs from other bacteria. ${ }^{20} \mathrm{To}$ examine LPS binding to OmpA $\cdot \mathrm{Skp}_{3}$ complexes, we gradually titrated these complexes with LPS and recorded the fluorescence spectra. Increased LPS binding to OmpA $\mathrm{Skp}_{3}$ resulted in an initial steep reduction of the fluorescence intensity (Figure 6(a), -) and in an initial steep increase of the wavelength of the intensity maximum $\lambda_{\max }$ (Figure $\left.6(\mathrm{~b}), 0\right)$ up to a molar ratio of about $3 \mathrm{LPS} /\left(\mathrm{OmpA} \cdot \mathrm{Skp}_{3}\right)$. At higher ratios, between 5 and $15 \mathrm{LPS} /\left(\mathrm{OmpA} \cdot \mathrm{Skp}_{3}\right)$, the decrease in intensity leveled off at $\sim 65 \%$ of the original intensity and the increase in $\lambda_{\max }$ approached $\sim 342 \mathrm{~nm}$. The LPS binding stoichiometry to $\mathrm{OmpA} \cdot \mathrm{Skp}_{3}$ is a far lower than the molar ratio of 25 LPS/OmpA observed previously for LPS binding to OmpA in absence of Skp. ${ }^{12}$ In absence of Skp, LPS binding to unfolded OmpA led to a fluorescence increase and a $\lambda_{\max }$ decrease to $\sim 335 \mathrm{~nm}$ (Figure 6, 0 ). These data suggest a different mode of interaction of LPS with the OmpA.Skp 3 complex compared to the interaction of LPS with OmpA alone, which is reflected in the fluorescence properties of OmpA.

To estimate the binding stoichiometry and the binding constant, we again assumed a simple mass action law with identical LPS-binding sites within

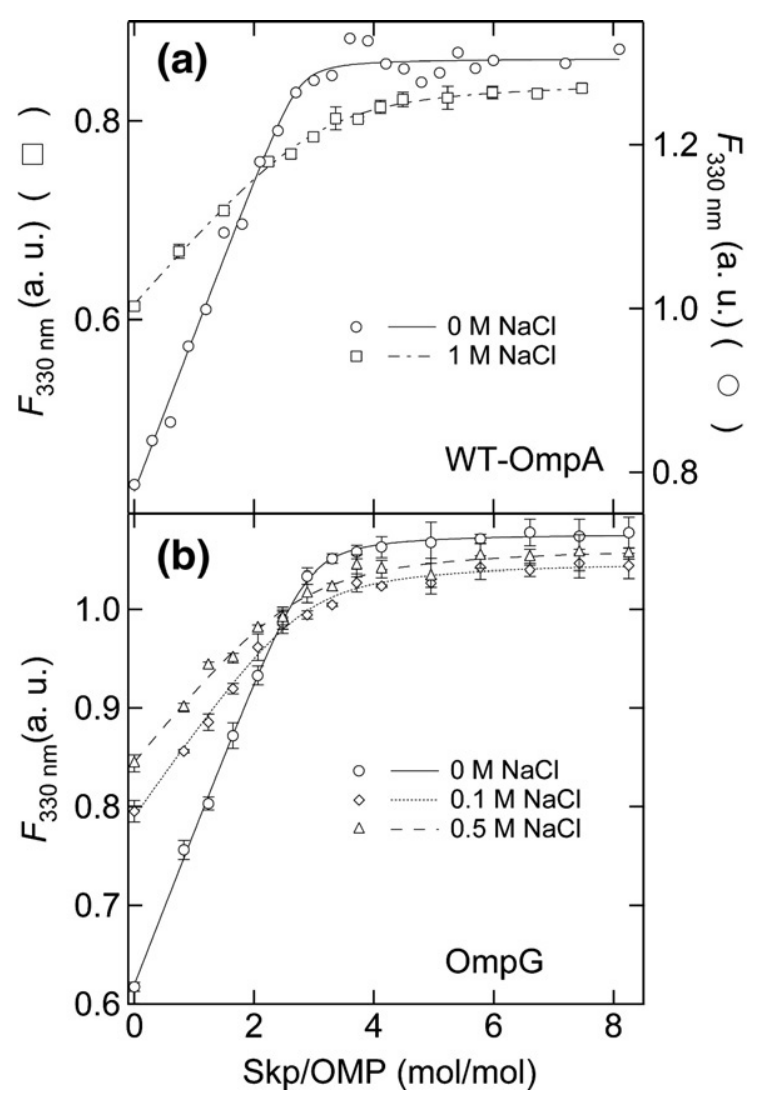

Figure 5. Skp binding to unfolded WT-OmpA and to unfolded OmpG at different concentrations of salt. (a) Fluorescence intensity increase upon addition of Skp to unfolded OmpA in the absence $(O)^{12}$ and in the presence of $1 \mathrm{M} \mathrm{NaCl}(\square)$. (b) Fluorescence intensity increase upon addition of Skp to unfolded OmpG in the absence of $\mathrm{NaCl}$ $(\bigcirc)$, in $0.1 \mathrm{M}(\triangle)$ and $0.5 \mathrm{M} \mathrm{NaCl}(\diamond)$. 


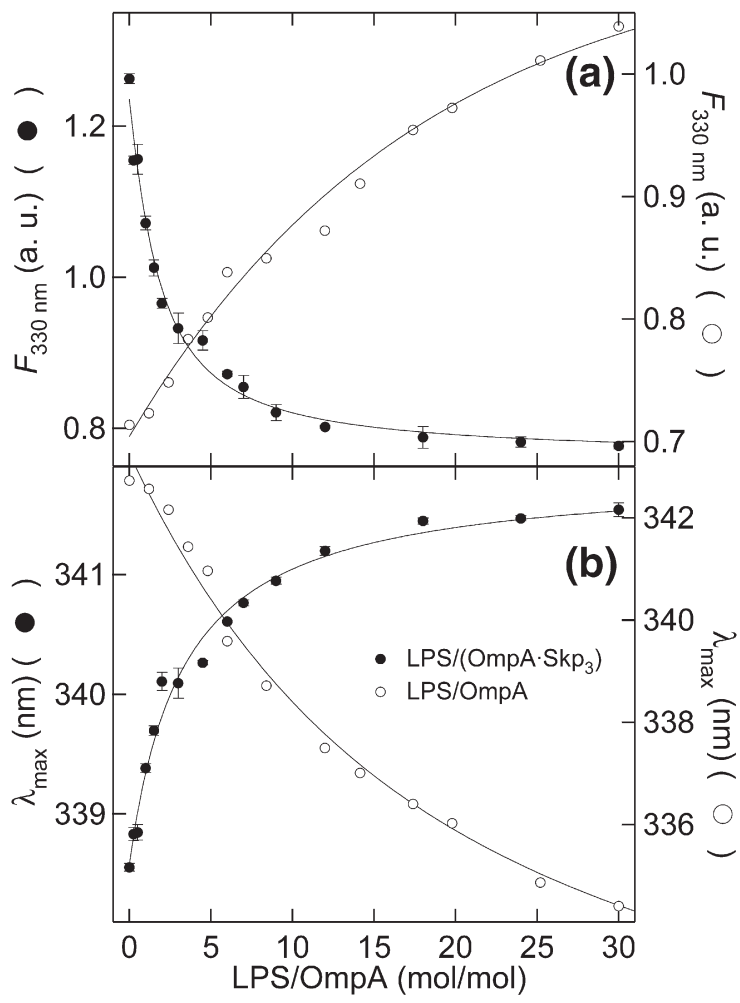

Figure 6. LPS binds to the OmpA $\mathrm{Skp}_{3}$ complex. (a) The intrinsic fluorescence intensity at $330 \mathrm{~nm}$ and (b) the wavelength of maximum fluorescence of the complex of OmpA and Skp were recorded after addition of LPS at molar LPS/(OmpA.Skp 3 ) ratios ranging from 0 to 30 . Three separate samples were measured at each concentration of LPS and an average value was calculated. $F_{330}$ and $\lambda_{\max }$ of OmpA after addition of LPS at molar LPS/OmpA ratios ranging from 0 to 30 are shown for comparison $(\mathrm{O})$. The data for LPS binding to WT-OmpA were taken from Bulieris et al. ${ }^{12}$

the $\mathrm{Skp}_{3} \cdot \mathrm{OmpA}$ complex. The fits to equation (2) suggested a low binding stoichiometry of $1.5( \pm 2)$ LPS/OmpA $\mathrm{Skp}_{3}$ and a dissociation constant of $K_{\mathrm{D}} \approx 500 \mathrm{nM}$. These were crude estimates, since LPS likely bound to both OmpA and Skp and therefore the assumption of equivalent binding sites may not be correct.

\section{Skp and LPS shield the tryptophan residues of OmpA against fluorescence quenching by acrylamide}

To test the average exposure of the five tryptophan residues of OmpA to the aqueous quencher acrylamide for both aqueous and Skp-bound forms of OmpA, we performed fluorescence quenching studies with acrylamide. We examined the effect of LPS on tryptophan accessibility for aqueous and Skp-bound OmpA (Figure 7). The fluorescence of aqueous OmpA was heavily quenched and a SternVolmer constant of $K_{S V}=6.0 \mathrm{M}^{-1}$ was calculated, consistent with previous results. ${ }^{32}$ In the presence of LPS, but in the absence of Skp, the Stern-Volmer constant is $4.5 \mathrm{M}^{-1}$, indicating LPS binding to aqueous OmpA, which partially shields it against quenching by acrylamide. In the presence of Skp, but in the absence of LPS, the quenching was weakest with a Stern-Volmer constant of $2.7 \mathrm{M}^{-1}$. In the presence of both Skp and LPS, the Stern-Volmer constant increased to $3.6 \mathrm{M}^{-1}$. Upon addition of LPS, the tryptophan residues of OmpA, which were wellshielded in the complex with Skp, became more accessible to the fluorescence quencher, but their fluorescence was still less quenched than for the aqueous OmpA in the absence of both Skp and LPS, or in the presence of LPS alone. These fluorescencequenching results supported the formation of a ternary complex between LPS, Skp and OmpA at low stoichiometries. They also indicated a conformational change in the OmpA.Skp ${ }_{3}$, complex, partially exposing hydrophobic tryptophan residues to the aqueous space.

\section{Discussion}

Our present results on the interactions of periplasmic Skp with OMPs provide detailed new insights into the properties and function of this membrane protein chaperone. After dilution of the urea, the aqueous forms of bacterial OMPs were bound by the Skp trimer with nanomolar affinity. Stable complexes were formed independent of the origin of the bacterial OMP. Binding did not occur with hVDAC1 from the OM of mitochondria,

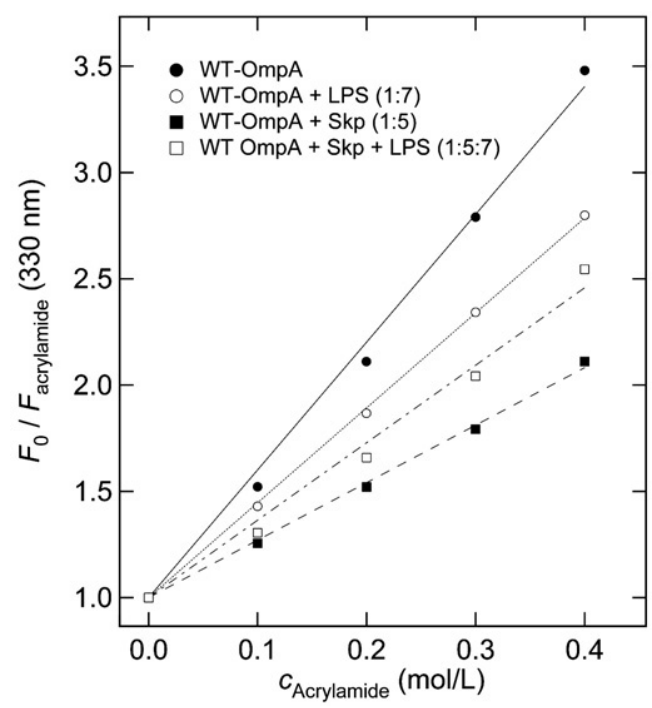

Figure 7. In the presence of LPS or Skp, the tryptophan residues of OmpA are less accessible to aqueous fluorescence quenchers. Fluorescence spectra of OmpA were recorded at different concentrations of the fluorescence quencher acrylamide to obtain the Stern-Volmer plots for OmpA in aqueous solution (-), in the presence of $S k p(\square)$, in the presence of LPS $(O)$ or in the presence of Skp and LPS ( $\square$ ). $F$ and $F_{0}$ are fluorescence intensities in the presence and in the absence of acrylamide, respectively. SternVolmer constants of $6.0 \mathrm{M}^{1}$ (OmpA), $4.5 \mathrm{M}^{1}$ (OmpA+ LPS), $2.7 \mathrm{M}^{1}$ (OmpA + Skp) and 3.6 $\mathrm{M}^{1}$ (OmpA + Skp+ LPS) were obtained from the slopes of linear fits. 
although hVDAC1 also forms a transmembrane $\beta$-barrel similar to bacterial OMPs. Independent of the size of their transmembrane domains, a 1:1 binding stoichiometry was observed for the bacterial OMPs. The binding affinity was highest for YaeT (Omp85), which is essential for the assembly of OMPs in bacteria. Both hydrophobic and electrostatic interactions contributed to the formation of $\mathrm{OMP} \cdot \mathrm{Skp}_{3}$ complexes. We further observed binding of the negatively charged LPS to OmpA.Skp complexes. LPS induced a partial exposure of OmpA to the aqueous phase. The LPS-induced topological change of the OmpA.Skp 3 complex could explain the facilitated folding of OmpA into lipid membranes in the presence of both Skp and LPS that was described earlier. ${ }^{12}$

\section{The Skp trimer binds bacterial OMPs at 1:1 stoichiometry}

The five bacterial OMPs (NalP, OmpG, OmpA, FomA, and YaeT) that ranged from $19 \mathrm{kDa}$ to $89 \mathrm{kDa}$ in molecular mass and from 8 to 14 in the number of their transmembrane strands, all bound $\mathrm{Skp}_{3}$ at 1:1 stoichiometry in aqueous solution after dilution of the denaturant. Other stoichiometries may be prevented because of steric constraints by the size of the proteins or because there is only one specific $\mathrm{Skp}_{3}$-binding site in each of the OMPs. The observed 1:1 stoichiometry for the interaction of the Skp trimer with OMPs does not completely exclude the formation of larger aggregates $\left(\mathrm{OMP} \cdot \mathrm{Skp}_{3}\right)_{n}$. Here, the $\mathrm{Skp}_{3}$ binding stoichiometries were determined for four bacterial OMPs that differed in size by a factor $>4$. It is far more likely that a large OMP would require two $\mathrm{Skp}_{3}$ for binding, leading to an OMP.Skp 6 complex, which is not observed here, rather than the smallest OMP would form an $\left(\mathrm{OMP} \cdot \mathrm{Skp}_{3}\right)_{2}$ complex. The facts that only one binding stoichiometry was obtained, independent of the size of the bound OMP, and that Skp alone forms trimers but not higher aggregates suggest strongly that the OMP.Skp 3 complexes do not form larger aggregates.

Skp of N. meningitides and Skp of F. nucleatum share about $22 \%$ and $23 \%$ sequence identity with Skp of E. coli. The differences between these Skp homologs are apparently not very important for the binding of OMPs, since NalP of N. meningitides and FomA of F. nucleatum both formed stable 1:1 complexes with E. coli $\mathrm{Skp}_{3}$, although the dissociation constant for NalP.Skp $\mathrm{Sp}_{3}$ was twice as high as those of OmpA and OmpG complexes at the same ionic strength (Table 1).

\section{Trimeric Skp binds OMPs with nanomolar affinity}

The binding affinities of $\mathrm{Skp}_{3}$ for various OMPs ranged from $0.3 \mathrm{nM}$ to $50 \mathrm{nM}$ (Table 2) at low ionic strength and were therefore about three orders of magnitude higher than the binding affinities of another periplasmic chaperone, SurA of E. coli, to
OmpG and $\mathrm{OmpF}^{33}$ In thermodynamic binding equilibria of OMPs in the presence of both, Skp and SurA, formation of OMP.Skp 3 complexes is favored over formation of OMP.SurA complexes and Skp should be the primary binding partner for OMPs if both Skp and SurA are present at the same sufficiently high concentration. It is possible that the expression levels of a single chaperone species are not high enough to solubilize all OMPs. Therefore, more than one species would be necessary to facilitate passage of all OMPs through the periplasm. Different binding affinities of the various chaperones in conjunction with regulated expression of chaperones and OMPs could lead to a preferred sequence of the assembly of OMPs into the OM.

It is interesting that the dissociation constant for complexes of $\mathrm{Skp}_{3}$ with YaeT is much smaller, by about two orders of magnitude, than the dissociation constants of all other OMP.Skp 3 complexes (Table 2), indicating that the binding affinity of $\mathrm{Skp}_{3}$ is strongest for YaeT. The periplasmic domain of YaeT, which binds polypeptides, ${ }^{26,34,35}$ may contribute to the increased stability. For folded YaeT in the outer membrane, the periplasmic domain may bind Skp to receive the unfolded OMP from the OMP.Skp 3 complex after passage through the periplasm. The possibility of selective binding of OMPs by the various periplasmic chaperones was proposed in a genetic study. ${ }^{36}$ Interestingly the $s k p$ gene follows the yaeT gene immediately downstream on the chromosome (e.g. see the online supplement to Voulhoux et al. ${ }^{25}$ ). Both Skp and YaeT are involved in the assembly pathway of OMPs. A preferred binding of Skp to YaeT in the periplasm and the possibility that expression levels of Skp may not be high enough to bind all the OMPs might explain why depletion of other chaperones like SurA led to reduced levels of OMPs like OmpA in the OM of E. coli. Each periplasmic chaperone, in addition to a general chaperone-function, may selectively bind a certain class of OMPs with higher affinities, similar to what is observed here for Skp binding to the aqueous form of YaeT. Preliminary data from our laboratory indicate that SurA can facilitate folding of OmpA into preformed phospholipid bilayers in the absence of both Skp and LPS, while Skp required the presence of LPS to facilitate folding of OmpA. ${ }^{12}$ This difference very likely is a consequence of the stability of the OMP.Skp 3 complexes, which is weakened by specific interactions with the negatively charged LPS. SurA binds OmpA with much weaker affinity, similar to $\mathrm{OmpG}$ and $\mathrm{OmpF}^{33}$ and the far less stable complexes do not require much activation to deliver the aqueous forms of OMPs to a membrane.

\section{Skp recognizes unfolded structures}

There are no homologs of Skp in eukaryotic cells and therefore it did not surprise us that neither BSA nor hVDAC1 bound to Skp. However, Skp prevented aggregation of lysozyme from hen eggs ${ }^{20}$ 
and promoted expression of soluble antibody fragments, ${ }^{10}$ indicating a more general role of $\mathrm{Skp}_{3}$ as a molecular chaperone. Soluble BSA was not bound by $\mathrm{Skp}_{3}$, because it folded too fast upon dilution of the urea. ${ }^{37}$ For the same reason, no $\mathrm{Skp}_{3}$ binding was observed for the soluble 154 residue periplasmic domain of OmpA, which folds independently of the transmembrane domain. ${ }^{8} \mathrm{Skp}$ recognizes unfolded and misfolded structures. Although hVDAC1 required a lipid bilayer for correct folding, our previous work indicated that this OMP rapidly developed large amounts of $\beta$-sheet secondary structure in aqueous solution, ${ }^{38}$ preventing the binding of Skp in our present study. In contrast, OmpA developed most of its $\beta$-sheet secondary structure not in aqueous solution, but when it inserted into the hydrophobic core of the membrane. ${ }^{39}$

\section{Skp interacts with hydrophobic residues of the transmembrane domain of OMPs}

$\mathrm{Skp}_{3}$ requires a significant content of non-native structure in its binding partner to form stable complexes. The binding partner must have either a high level of conformational flexibility or surfaceexposed residues that are inaccessible when folded, or both. For folded $\beta$-barrels of transmembrane proteins, the polar residues are oriented towards the barrel lumen, while hydrophobic residues form the outer surface. The hydrophobic residues include the fluorescent tryptophan residues that are exposed to the lipids predominantly in their membraneinserted, folded forms. The aqueous forms of OMPs, which are obtained after dilution of the urea, have, at least partially, an inside-out conformation, in which hydrophobic residues are predominantly buried inside a collapsed conformation, while the charged and polar residues are surface-oriented and water-exposed. The fluorescence data obtained for the bacterial OMPs indicated that the tryptophan residues are partially shielded from the aqueous space in the hydrophobically collapsed OMPs. In OMP.Skp ${ }_{3}$ complexes, they are much less accessible, which is obvious from fluorescence intensity increases $\left(F_{\mathrm{C}} / F_{\mathrm{AQ}}\right)$, shifts in $\lambda_{\max }$ of the spectra, and from the quenching experiments with acrylamide. The tryptophan residues of the OMPs interact with hydrophobic residues of $\mathrm{Skp}_{3}$. An interpretation could be that $\mathrm{Skp}_{3}$ binding turns around the partial inside-out conformation of the aqueous forms of the OMPs, requiring their conformational flexibility. This could then be consistent with a lack of Skp binding to hVDAC1, which developed most of the $\beta$-sheet structure already in aqueous solution. The exposed hydrophobic surface of a largely preformed antiparallel $\beta$-sheet of hVDAC1 could be too rigid to interface well with the hydrophobic surface of Skp inside the Skp tentacle basket.

The flexible three-pronged forceps-like structure of $\mathrm{Skp}_{3}$ suggests that OMPs are bound inside the basket defined by the six $\alpha$-helices of $\mathrm{Skp}_{3}$ (Figure 8 ). As pointed out previously, ${ }^{19,20}$ the abundance of positively charged residues at the end of the tentacles and the flexibility of the tentacles likely ensure that the inside of the tentacle domain is accessible in solution. Charged and hydrophobic areas form patches over the entire surface of the tentacle domain, which has a high positive net charge (Figure 8). Inside the tentacle basket, there are hydrophobic patches formed between the helices of each monomer. Two of them are composed of residues F13, A17, V22, and L26 or of residues F30, A34, L37, F74, and F81. These hydrophobic residues also form the interface between the two helices of the Skp monomer (Figure $8(c))$. It is quite possible that hydrophobic residues of the transmembrane domains of the OMPs are bound at these locations.

\section{Skp binds OMPs by electrostatic interactions}

$\mathrm{Skp}_{3}$ bound $\mathrm{OmpA}$ at a $\mathrm{pH}$ between the isoelectric points of OmpA and Skp, i.e. when OmpA was negatively charged and Skp positively charged. $\mathrm{NalP}$ (or FomA) binding to $\mathrm{Skp}_{3}$ was observed down to $\mathrm{pH}$, at which both NalP and FomA are positively charged. Since the positively charged $\mathrm{Skp}_{3}$ still bound, complex formation may be caused either by hydrophobic interactions or by chargecharge interactions with specific regions of NalP (or FomA), in which the residues aspartate and glutamate dominate. $\mathrm{Skp}_{3}$ binding to NalP (or FomA) was not observed when $\mathrm{Skp}_{3}$ lost its positive net charge at very basic $\mathrm{pH}$. Therefore, we concluded that a partially asymmetric distribution of positive and negative charges in OMPs such as NalP, results in the binding of positively charged $\mathrm{Skp}_{3}$ at regions of the NalP or FomA, where their negative charges dominate. An asymmetric charge distribution in $\mathrm{NalP}$ and FomA also explains the $\mathrm{pH}$-dependence of the fluorescence spectra of NalP and FomA, which indicated strong conformation changes in both OMPs due to protonation below $\mathrm{pH} 6$ and $\mathrm{pH} 7$, i.e. well below their calculated isoelectric points (Figure 3(b) and (c)). Below this $\mathrm{pH}$ region, NalP and FomA become more compact, as indicated by increased fluorescence and by shifts in $\lambda_{\max }$ towards shorter wavelengths. This is very likely a consequence of reduced charge-charge repulsion and reduced hydration of side-chains upon neutralization by protonation.

The periplasmic turns are regions of the OMPs that are rich in negatively charged aspartate and glutamate residues (Table 3). Similarly, some outer loops of certain OMPs may also carry a negative net charge although they are often positively charged. For instance, FhuA displayed selectivity for negatively charged lipids in lipid bilayers, because positive charges dominated in the outer loops near the lipid headgroup region. ${ }^{40}$ The loops of NalP have a net charge of +2 , while the turns have a net charge of -3 . In the OM, the OMP loops face the negatively charged LPS. The loops may contain positively charged binding sites for the phosphate groups of LPS. In the short periplasmic turns, 
(a)

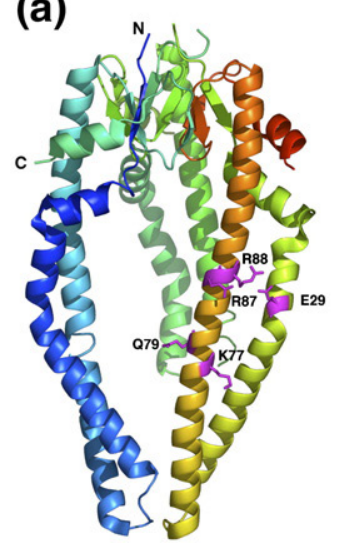

(b)

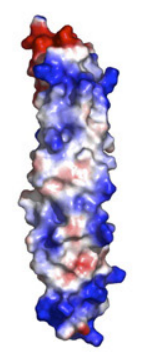

A

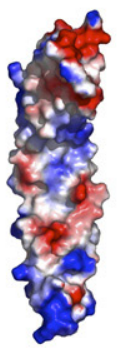

B

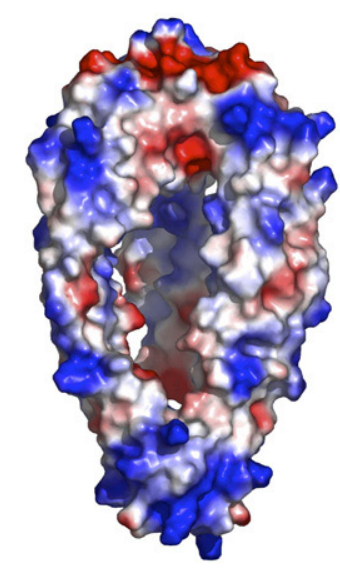

(c)

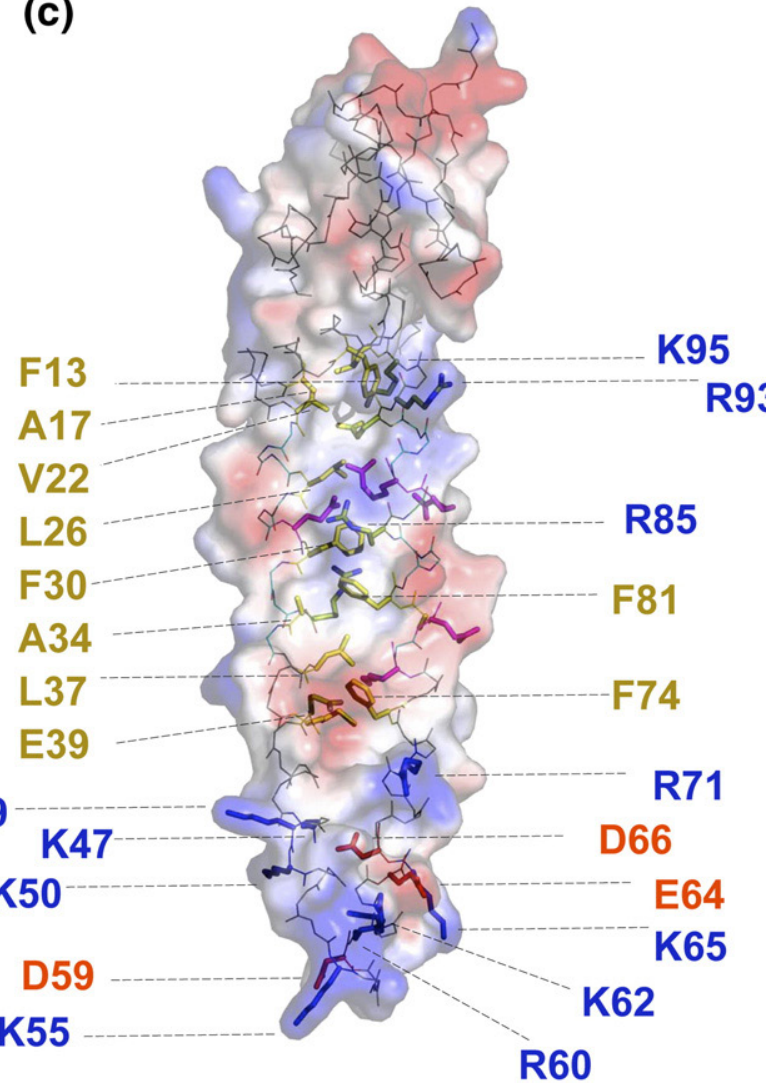

Figure 8. Crystal structure of Skp (PDB1SG $2^{19}$ and $1 \mathrm{U}_{2} \mathrm{M}^{20}$ ). (a) The Skp trimer is composed of a tentacle domain and an association domain. The association domain contains a nine-stranded $\beta$-barrel. This domain is surrounded by three pairs of $\alpha$-helices that are about $65 \AA$ long and form the tentacle domain. Each 141 residue Skp monomer contains a predicted LPS-binding site composed of residues K77, R87, and R88. The spatial orientation of these residues matches a conserved LPS-binding motif previously described for the OMP FhuA ${ }^{41}$ at a root-mean-square (rms) deviation of $1.75 \AA{ }^{20}$ The surface of the trimer is strongly positively charged, because each monomer contains 28 lysine and arginine residues, but only 19 aspartate and glutamate residues. (b) The charged residues are distributed asymmetrically, and most of the negatively charged aspartate and glutamate residues are found in the association domain, while the positively charged residues are found at the other end at the tips of the tentacle domain, leading to a large dipole moment of $\sim 3700$ Debye $(770 \mathrm{e} \AA) .{ }^{19}$ (c) The tip of each tentacle contains eight positively charged residues (K and L), but only three negatively charged residues ( $\mathrm{D}$ and $\mathrm{E}$ ) and is the region of the highest positive charge density.

negatively charged residues are found sometimes adjacent in pairs, which increases the local charge density and the attraction to the positively charged surface of Skp. It is expected that in the inside-out water-collapsed state of OMPs, the charged residues would be water-exposed at the OMP surface. Negatively charged turn or loop regions may therefore interact with the most basic regions of $\mathrm{Skp}_{3}$. The Skp trimer has the highest positive charge density at the tips of the three tentacles (Figure 8) which each contain eight basic (six lysine and two arginine), but only three acidic residues (Figure 8(c)). Possibly, these three tip regions are sites in $\mathrm{Skp}_{3}$ that contribute most strongly to the observed electrostatic interactions, in particular if the hydrophobic residues of the transmembrane strands are bound inside the tentacle basket as suggested. ${ }^{19,20}$ Interactions of the positively charged tentacle tips of $\mathrm{Skp}_{3}$ with negatively charged and polar residues of the periplasmic turns in combination with hydrophobic interactions of the strand regions of the OMPs inside the $\mathrm{Skp}_{3}$ tentacle basket could then also mean that the OMPs adopt some kind of orientation upon binding to $\mathrm{Skp}_{3}$.

\section{LPS modulates the topology of the OmpA.Skp 3 complex}

It has been shown that Skp alone inhibits folding of OmpA and that LPS reverses the retarding effect of $\mathrm{Skp}_{3}$ by interacting with the OmpA.Skp $\mathrm{Op}_{3}$ complex. ${ }^{12}$ Our acrylamide quenching experiments indicated that LPS changes the topology of the OmpA $\cdot \mathrm{Skp}_{3}$ complex and partially exposes the fluorescent tryptophan residues of OmpA to the aqueous environment (Figures 6 and 7) even when present at only low molar ratios of LPS per OmpA $\cdot \mathrm{Skp}_{3}$ complex. The stoichiometry estimated for LPS binding to OmpA $\cdot \mathrm{Skp}_{3}$ complexes (Figure 6) corresponds well to our previous observations of optimal OmpA folding kinetics and folding yields at an LPS content of 2-7 mol per mol OmpA.Skp complex. ${ }^{12}$ The fluorescence quenching experiments with acrylamide suggested that LPS is not able to 
Table 3. Acidic and basic residues in the periplasmic turns of some OMPs

\begin{tabular}{lccrrrrrc}
\hline OMP & PDB & $Z_{\mathrm{TM} \beta \mathrm{s}}{ }^{\mathrm{a}}$ & $Z_{\mathrm{T}}^{\mathrm{b}}$ & $D^{\mathrm{c}}$ & $E^{\mathrm{c}}$ & $K^{\mathrm{c}}$ & $R^{\mathrm{c}}$ & T charge $^{\mathrm{d}}$ \\
\hline OmpA & 1BXW & 8 & 3 & 2 & 1 & 0 & 0 & -3 \\
NalP & 1UYN & 12 & 5 & 2 & 1 & 0 & 0 & -3 \\
OmpG & 2IWW & 14 & 6 & 5 & 1 & 0 & 0 & -6 \\
OmpW & 2F1V & 8 & 3 & 3 & 0 & 0 & 1 & -2 \\
OmpT & $1 \mathrm{I} 78$ & 10 & 4 & 1 & 2 & 0 & 0 & -3 \\
FadL & 1T16 & 14 & 6 & 6 & 0 & 0 & 2 & -4 \\
OmpF & 2OmF & 16 & 7 & 4 & 1 & 1 & 0 & -5 \\
LamB & 1MAL & 18 & 8 & 3 & 2 & 1 & 0 & -4 \\
FhuA & 2FCP & 22 & 10 & 11 & 1 & 2 & 2 & -8 \\
\hline
\end{tabular}

a Number of transmembrane $\beta$ strands of the OMP.

b Number of periplasmic $\beta$ turns of the OMP.

c Numbers of aspartate (D), glutamate (E), lysine (K), and arginine residues $(R)$ in the periplasmic turns of these OMPs.

d Total charge of all turns.

d Translocator domain of NalP, residues 7761083

completely displace $\mathrm{Skp}_{3}$ from OmpA (Figure 7). The LPS core region contains two phosphate and two diphosphate groups, which are negatively charged. Since the outer surface of Skp is very polar and positively charged in the tentacle domain, the binding of individual LPS molecules to $\mathrm{Skp}_{3}$ involves electrostatic interactions with the OmpA'Skp $\mathrm{Sm}_{3}$ complex. Such interactions would be expected in the predicted LPS binding-site of Skp that contains two positively charged arginine and lysine residues, 20,41 or in the tip regions of Skp, or both. Although covalent bonds are not formed, the interaction of LPS with OmpA $\cdot \mathrm{Skp}_{3}$ is exergonic and provides a significant amount of free energy for a conformation change in the complex. This weakens the interaction of $\mathrm{Skp}_{3}$ with bound OmpA and results in a topology change of the complex that partially exposes the tryptophan residues of OmpA to the aqueous environment. In the fluorescence experiments, this was reflected by a decrease of intensity and by an increase of $\lambda_{\max }$ of the tryptophan fluorescence of OmpA upon LPS binding (Figure 6). In the proximity of a lipid bilayer, the LPS-induced topology change led to the release of the OMP from the complex for insertion and folding into the membrane. ${ }^{12}$ In conclusion, our results suggest that charge-charge interactions are necessary for binding and release of OMPs by the periplasmic chaperone $\mathrm{Skp}_{3}$.

\section{Materials and Methods}

\section{Purification of Skp, WT-OmpA, TMD-OmpA, FomA and hVDAC1}

Wild-type OmpA, ${ }^{12}$ TMD-OmpA, ${ }^{42}$ FomA, ${ }^{30}$ and hVDAC $1^{38}$ were over-expressed and purified from E. coli in their unfolded forms as described. Skp was isolated from E. coli as described. ${ }^{12}$

\section{Purification of OmpG, NalP and YaeT}

The ompG gene (lacking the nucleotide sequence encoding the signal peptide) was amplified by PCR $\left(60{ }^{\circ} \mathrm{C}\right.$ annealing temperature) using $50 \mathrm{ng}$ of $\mathrm{E}$. coli MG1655 genomic DNA as template and the primers

\section{5'-TAGGGCCATATGGAGGAAAGGAACGACTGG-3' 5'-CTACTCGAGTCAAAGCTTGAACGAGTAATT- TACGCCG-3'}

The PCR product was cloned into the $\mathrm{pET} 29 \mathrm{~b}$ vector (Novagen) using the NdeI/Xhol restriction sites, yielding pET29OmpGm2. Plasmid pET29OmpGm2 was transformed into E. coli BL21 (DE3) (Stratagene) to express OmpG in the form of inclusion bodies. A culture $(20 \mathrm{ml})$ was grown for $18 \mathrm{~h}$ and used to inoculate 21 of LB medium. After $3 \mathrm{~h}$, IPTG was added to a final concentration of $0.1 \mathrm{mM}$. After 4 to $6 \mathrm{~h}$ of induction, cells were harvested by centrifugation for $30 \mathrm{~min}$ at $1500 \mathrm{~g}$ at $4{ }^{\circ} \mathrm{C}$. The wet cell paste was resuspended in $40 \mathrm{ml}$ of $20 \mathrm{mM}$ Tris $\mathrm{HCl}(\mathrm{pH} 8.0), 0.1 \%(\mathrm{v} / \mathrm{v}) \beta$-mercaptoethanol while cooling in an ice/water bath. Lysozyme was added to a concentration of $50 \mu \mathrm{g} / \mathrm{ml}$ and the mixture was stirred for $30 \mathrm{~min}$ at $22{ }^{\circ} \mathrm{C}$. The solution was then sonicated for 30 min using a Branson ultrasonifier W-450D (20\% power, $50 \%$ pulse cycle) with a macrotip while cooling in an ice/ water bath. The buffer and soluble proteins were removed by centrifugation at $3000 \mathrm{~g}$ for $30 \mathrm{~min}$ at $4{ }^{\circ} \mathrm{C}$. The pellet was washed in $20 \mathrm{ml}$ of $20 \mathrm{mM}$ Tris $\mathrm{HCl}(\mathrm{pH}$ $8.0), 0.1 \% \beta$-mercaptoethanol, $1 \mathrm{M}$ urea. The supernatant was removed by centrifugation at $5000 \mathrm{~g}$ for $30 \mathrm{~min}$ at $25{ }^{\circ} \mathrm{C}$. The pellet was then dissolved in $40 \mathrm{ml}$ of $20 \mathrm{mM}$ Tris $\mathrm{HCl}(\mathrm{pH} 8.0), 0.1 \% \beta$-mercaptoethanol, $8 \mathrm{M}$ urea. The solution was loaded onto a Q-Sepharose FF column (Amersham) and the proteins were eluted by a $\mathrm{NaCl}$

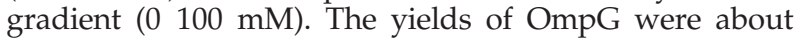
$50 \mathrm{mg}$ from 11 of culture.

Restriction sites for NcoI and BamHI were used for cloning yaeT into the $\mathrm{pET} 15 \mathrm{~b}$ vector (Novagen). The resulting plasmid, pET15 EcOMP85, was transformed into E. coli BL21 (DE3). His ${ }_{6}$-tagged YaeT was expressed in the form of inclusion bodies in E. coli. YaeT was purified from these inclusion bodies as described for OmpG.

For expression of the translocator domain of NalP (residues D776 to F1083 of NalP), plasmid pPU320 was transformed into E. coli BL21 (DE3) (Stratagene) and the NalP protein was purified as described. ${ }^{24}$

\section{Purification of R-LPS}

E. coli rough mutant F576 was cultivated as described, ${ }^{43}$ and its LPS (R2 core type, $M \approx 3900 \mathrm{~g} / \mathrm{mol}$ ) was isolated as described. ${ }^{44}$

\section{Fluorescence spectroscopy}

Fluorescence spectra were recorded as described ${ }^{12}$ on a Spex Fluorolog-3 spectrofluorimeter with double monochromators in the excitation and emission pathways. The excitation wavelength was $295 \mathrm{~nm}$, and the bandwidths of the excitation monochromators were $2.5 \mathrm{~nm}$. The bandwidths of the emission monochromators were $5 \mathrm{~nm}$. The integration time was $0.05 \mathrm{~s}$, and an increment of $0.5 \mathrm{~nm}$ was used to scan spectra in the range of $310380 \mathrm{~nm}$. Background intensities of Skp in the absence of OMPs were subtracted. Emission maxima of the spectra were calculated by fitting a lognormal distribution to the experimental spectra using Igor Pro 6.0 (WaveMetrics, Oregon). ${ }^{45}$ All experiments were done at $25^{\circ} \mathrm{C}$. Each experiment was performed three times, and average intensities and emission maxima were calculated. 


\section{Binding of Skp to OMPs monitored by fluorescence spectroscopy}

To monitor binding of Skp to OMPs, the background spectrum of Skp at a selected concentration was first recorded in $990 \mu \mathrm{l}$ of urea-free $10 \mathrm{mM}$ glycine buffer $(\mathrm{pH}$ 9.0). The concentrations of Skp were chosen to obtain Skp/ OMP molar ratios ranging from 0 to 8 . Then $10 \mu \mathrm{l}$ of a stock solution of an unfolded OMP in $8 \mathrm{M}$ urea was added and the fluorescence spectrum was recorded after $30 \mathrm{~s}$ of incubation. The background spectra of Skp were subtracted. Separate sets of fluorescence spectra were recorded for each OMP at final concentrations of $0.37 \mu \mathrm{M}$ OmpG, $0.16 \mu \mathrm{M}$ YaeT, $0.55 \mu \mathrm{M}$ NalP, and $0.83 \mu \mathrm{M}$ TMD-OmpA. In these experiments, the OMPs were diluted from concentrated stock solutions to reduce the urea concentration 100-fold.

Binding functions were fit to the experimental data assuming one class of identical binding sites in each OMP. In this case, the average concentration of bound Skp is given by: ${ }^{46}$

$$
[\mathrm{B}] /\left[{ }^{\mathrm{t}} \mathrm{OMP}\right]=n K_{\mathrm{ass}}[\mathrm{F}] /\left(1+K_{\mathrm{ass}}[\mathrm{F}]\right)
$$

where $n$ is the number of binding sites, $K_{\text {ass }}$ is the association constant, $\left[{ }^{t} \mathrm{OMP}\right]$ is the total concentration of the OMP, and $[\mathrm{F}]$ is the concentration of the free ligand. Substitution of the free ligand with the total ligand concentration, $\left[\mathrm{L}_{0}\right]=[\mathrm{B}]+[\mathrm{F}]$ and some rearrangements led to:

$$
\begin{aligned}
{[\mathrm{B}]=} & 0.5\left\{\mathrm{~K}_{\mathrm{ass}}{ }^{-1}+\left[\mathrm{L}_{0}\right]+n\left[{ }^{\mathrm{t}} \mathrm{OMP}\right]\right. \\
& \left.\left(\left(K_{\mathrm{ass}}{ }^{-1}+\left[\mathrm{L}_{0}\right]+n\left[{ }^{\mathrm{t}} \mathrm{OMP}\right]\right)^{2} \quad 4 n\left[{ }^{\mathrm{t}} \mathrm{OMP}\right]\left[\mathrm{L}_{0}\right]\right)^{1 / 2}\right\}
\end{aligned}
$$

where $[\mathrm{B}]$ and $\left[{ }^{\mathrm{t}} \mathrm{OMP}\right][\mathrm{B}]$ are the concentrations of bound and free OMP, respectively. The fluorescence signal of the $\mathrm{OMP}$ in binding experiments is a linear combination of the concentrations of bound and free OMP:

$$
F=f_{\mathrm{b}}[\mathrm{B}]+f_{\mathrm{f}}\left(\left[{ }^{\mathrm{t}} \mathrm{OMP}\right]\right.
$$

where $f_{\mathrm{b}}$ and $f_{\mathrm{f}}$ are constants that describe the fluorescence contributions of bound and free OMP, respectively.

\section{Skp binding to unfolded OMPs as a function of $\mathrm{pH}$}

To determine whether binding of Skp is $\mathrm{pH}$-dependent, spectra of OMPs were recorded as described above, but in other buffers $(10 \mathrm{mM})$ at various $\mathrm{pH}$ values: citrate, $\mathrm{pH} 3$ 6; Hepes, pH 7; Tris, pH 8; glycine, pH 9 10; and Caps, pH 11. A fivefold molar excess of Skp was used. Background spectra of Skp were recorded and subtracted. The OMP concentrations were $0.43 \mu \mathrm{M}$ OmpA, $0.47 \mu \mathrm{M}$ NalP, $0.65 \mu \mathrm{M}$ hVDAC1, and $0.20 \mu \mathrm{M}$ FomA.

\section{Skp binding to unfolded OmpA or to unfolded OmpG as a function of ionic strength}

To determine binding at different ionic strengths, experiments were performed in $10 \mathrm{mM}$ Tris ( $\mathrm{pH} 8.0$ ), containing either $0,0.1 \mathrm{M}, 0.2 \mathrm{M}, 0.5 \mathrm{M}$ or $1 \mathrm{M} \mathrm{NaCl}$. Titrations with Skp were done with $0.43 \mu \mathrm{M}$ OmpA and $0.37 \mu \mathrm{M}$ OmpG as described above.

\section{Dynamic light-scattering}

The hydrodynamic radius and particle mass of Skp in solution were measured by dynamic light scattering using a Dynapro instrument (Wyatt Technology Corp.). Solutions of Skp $(2 \mathrm{~g} / \mathrm{l})$ in $12 \mu \mathrm{l}$ of buffer were first passed through aluminum oxide filters (Whatman) of $0.02 \mu \mathrm{m}$ pore size. The intensity of the scattered light was measured and the hydrodynamic radius $\left(R_{\mathrm{H}}\right)$ was analyzed using the software Dynamics, Ver. $6 . R_{\mathrm{H}}$ was calculated from the theoretical hydrodynamic radius of an ideally spherical particle, $R_{\mathrm{HTH}}$, and the ratio of the frictional coefficients, $f / f_{0}$ for a hydrated versus nonhydrated sphere according to:

$$
R_{\mathrm{H}} / R_{\mathrm{HTH}}=f / f_{0}
$$

The molecular mass of the Skp particles can be calculated from the hydrodynamic radius:

$$
M=(4 / 3) \pi\left[\left(f_{0} / f\right) R_{\mathrm{H}}\right]^{3} /\left[\left(V_{P}+H_{\mathrm{AQ}}\right) N_{A}\right]
$$

where $V_{\mathrm{p}}$ is the specific volume of the particle (for a protein, an average of $0.73 \mathrm{~cm}^{3} / \mathrm{g}$ is normally assumed ${ }^{47}$ ), $H_{\mathrm{AQ}}$ is the hydration volume of the protein (typically assumed to be $0.35 \mathrm{~cm}^{3} / \mathrm{g}$ protein). For a spherical protein, $f / f_{0}=1$.2. For $S k p$, which resembles a prolate ellipsoid, the Perrin factor is 1.02, leading to $f / f_{0}=1.22 N_{A}$ is Avogadro's number.

\section{CD spectroscopy}

Far-UV CD spectra were recorded at room temperature with a Jasco 715 CD spectrometer (Jasco, Tokyo, Japan) using a $0.5 \mathrm{~mm}$ path length cuvette. Three scans were accumulated from $190250 \mathrm{~nm}$ with a response time of $8 \mathrm{~s}$, a bandwidth of $1 \mathrm{~nm}$ and a scan speed of $50 \mathrm{~nm} / \mathrm{min}$. Background spectra without Skp were subtracted. The concentration of Skp $(30 \mu \mathrm{M})$ was determined for each sample using the method of Lowry. ${ }^{48}$ The recorded CD spectra were normalized to the mean residue molar ellipticity $[\Theta](\lambda)$, given by:

$$
[\Theta](\lambda)=100 \frac{\Theta(\lambda)}{c \cdot n \cdot l}
$$

where $l$ is the path length of the cuvette in $\mathrm{cm}, \Theta(\lambda)$ is the recorded ellipticity in degrees at wavelength $\lambda, c$ is the concentration in $\mathrm{mol} / \mathrm{l}$, and $n$ is the number of amino acid residues of $\operatorname{Skp}(141)$.

\section{LPS binding to the OmpA.Skp ${ }_{3}$ complex}

The fluorescence spectra of OmpA $\cdot \mathrm{Skp}_{3}$ complexes were recorded in the absence and in the presence of increasing concentrations of LPS in $10 \mathrm{mM}$ Tris $\mathrm{HCl}$ (pH 8.0). The concentrations of OmpA and Skp were $0.43 \mu \mathrm{M}$ and $1.3 \mu \mathrm{M}$, respectively. The LPS concentration ranged from 0 to $13 \mu \mathrm{M}$. Background spectra of Skp were subtracted.

\section{Fluorescence quenching experiments with acrylamide}

Acrylamide quenching experiments were performed in $10 \mathrm{mM}$ Tris $\mathrm{HCl}(\mathrm{pH} 8.0)$ with $0,0.1 \mathrm{M}, 0.2 \mathrm{M}, 0.3 \mathrm{M}$ and $0.4 \mathrm{M}$ acrylamide. The concentrations of OmpA, Skp and LPS were $0.85 \mu \mathrm{M}, 4.25 \mu \mathrm{M}$ and $6 \mu \mathrm{M}$, respectively. The data were fit to the Stern-Volmer function: $F_{0} / F=1+K_{\mathrm{sv}}[\mathrm{Q}] . K_{\mathrm{sv}}$ is the Stern-Volmer constant and $[Q]$ is the concentration of acrylamide. $F$ was corrected for the inner filter effect for absorption to obtain $F_{\text {corr }}=F_{\text {meas }} \times 10^{1 / 2 \times 0.55 \times[Q]}$. 


\section{Acknowledgements}

This work was supported by grants KL1024/4-1 and $4 / 2$ from the Deutsche Forschungsgemeinschaft to J.H.K. We are grateful to Dr Peter v. Ulsen, University of Utrecht, for the generous gift of plasmid pPU320 encoding the translocator domain of NalP and to Alexander Brosig, University of Konstanz, for his help with the light-scattering experiments.

\section{References}

1. Kleinschmidt, J. H. (2007). Assembly of integral membrane proteins from the periplasm into the outer membrane. In The Periplasm, Ehrmann, M., ed), pp. 30 66, ASM Press, Washington, DC.

2. Alba, B. M. \& Gross, C. A. (2004). Regulation of the Escherichia coli $\sigma^{\mathrm{E}}$-dependent envelope stress response. Mol. Microbiol. 52, 613619.

3. Ehrmann, M. \& Clausen, T. (2004). Proteolysis as a regulatory mechanism. Annu. Rev. Genet. 38, 709724.

4. Duguay, A. R. \& Silhavy, T. J. (2004). Quality control in the bacterial periplasm. Biochim. Biophys. Acta, 1694, 121134

5. Dartigalongue, C., Missiakas, D. \& Raina, S. (2001). Characterization of the Escherichia coli $\sigma^{\mathrm{E}}$-regulon. J. Biol. Chem. 276, 2086620875.

6. Bitto, E. \& McKay, D. B. (2003). The periplasmic molecular chaperone protein SurA binds a peptide motif that is characteristic of integral outer membrane proteins. J. Biol. Chem. 278, 4931649322.

7. Hennecke, G., Nolte, J., Volkmer-Engert, R., SchneiderMergener, J. \& Behrens, S. (2005). The periplasmic chaperone SurA exploits two features characteristic of integral outer membrane proteins for selective substrate recognition. J. Biol. Chem. 280, 2354023548.

8. Chen, R. \& Henning, U. (1996). A periplasmic protein (Skp) of Escherichia coli selectively binds a class of outer membrane proteins. Mol. Microbiol. 19, 12871294.

9. Schäfer, U., Beck, K. \& Müller, M. (1999). Skp, a molecular chaperone of gram-negative bacteria, is required for the formation of soluble periplasmic intermediates of outer membrane proteins. J. Biol. Chem. 274, 2456724574.

10. Bothmann, H. \& Plückthun, A. (1998). Selection for a periplasmic factor improving phage display and functional periplasmic expression. Nature Biotechnol. 16, 376380 .

11. Wülfing, C. \& Plückthun, A. (1994). Protein folding in the periplasm of Escherichia coli. Mol. Microbiol. 12, 685692.

12. Bulieris, P. V., Behrens, S., Holst, O. \& Kleinschmidt, J. H. (2003). Folding and insertion of the outer membrane protein OmpA is assisted by the chaperone Skp and by lipopolysaccharide. J. Biol. Chem. 278, 90929099.

13. Kleinschmidt, J. H. (2006). Folding kinetics of the outer membrane proteins OmpA and FomA into phospholipid bilayers. Chem. Phys. Lipids, 141, 3047.

14. Roy, A. M. \& Coleman, J. (1994). Mutations in firA, encoding the second acyltransferase in lipopolysaccharide biosynthesis, affect multiple steps in lipopolysaccharide biosynthesis. J. Bacteriol. 176, 16391646.

15. Thome, B. M., Hoffschulte, H. K., Schiltz, E. \& Müller, M. (1990). A protein with sequence identity to Skp
(FirA) supports protein translocation into plasma membrane vesicles of Escherichia coli. FEBS Letters, 269, 113116

16. de Cock, H., Brandenburg, K., Wiese, A., Holst, O. \& Seydel, U. (1999). Non-lamellar structure and negative charges of lipopolysaccharides required for efficient folding of outer membrane protein PhoE of Escherichia coli. J. Biol. Chem. 274, 51145119.

17. Freudl, R., Schwarz, H., Stierhof, Y. D., Gamon, K., Hindennach, I. \& Henning, U. (1986). An outer membrane protein $(\mathrm{OmpA})$ of Escherichia coli $\mathrm{K}-12$ undergoes a conformational change during export. J. Biol. Chem. 261, 1135511361.

18. Schlapschy, M., Dommel, M. K., Hadian, K., Fogarasi, M., Korndörfer, I. P. \& Skerra, A. (2004). The periplasmic E. coli chaperone Skp is a trimer in solution: biophysical and preliminary crystallographic characterization. Biol. Chem. 385, 137143.

19. Korndörfer, I. P., Dommel, M. K. \& Skerra, A. (2004). Structure of the periplasmic chaperone Skp suggests functional similarity with cytosolic chaperones despite differing architecture. Nature Struct. Mol. Biol. 11, 10151020.

20. Walton, T. A. \& Sousa, M. C. (2004). Crystal structure of Skp, a prefoldin-like chaperone that protects soluble and membrane proteins from aggregation. Mol. Cell, 15, 367374.

21. Ferguson, A. D., Hofmann, E., Coulton, J. W., Diederichs, K. \& Welte, W. (1998). Siderophoremediated iron transport: crystal structure of FhuA with bound lipopolysaccharide. Science, 282, 22152220 .

22. Yildiz, O., Vinothkumar, K. R., Goswami, P. \& Kühlbrandt, W. (2006). Structure of the monomeric outer-membrane porin OmpG in the open and closed conformation. EMBO J. 25, 37023713.

23. Subbarao, G. V. \& van den Berg, B. (2006). Crystal structure of the monomeric porin OmpG. J. Mol. Biol. 360, 750759 .

24. Oomen, C. J., Van Ulsen, P., Van Gelder, P., Feijen, M., Tommassen, J. \& Gros, P. (2004). Structure of the translocator domain of a bacterial autotransporter. EMBO J. 23, 12571266.

25. Voulhoux, R., Bos, M. P., Geurtsen, J., Mols, M. \& Tommassen, J. (2003). Role of a highly conserved bacterial protein in outer membrane protein assembly. Science, 299, 262265.

26. Clantin, B., Delattre, A. S., Rucktooa, P., Saint, N., Meli, A. C., Locht, C. et al. (2007). Structure of the membrane protein FhaC: a member of the Omp85TpsB transporter superfamily. Science, 317, 957961.

27. Werner, J. \& Misra, R. (2005). YaeT (Omp85) affects the assembly of lipid-dependent and lipid-independent outer membrane proteins of Escherichia coli. Mol. Microbiol. 57, 14501459.

28. Doerrler, W. T. \& Raetz, C. R. (2005). Loss of outer membrane proteins without inhibition of lipid export in an Escherichia coli YaeT mutant. J. Biol. Chem. 280, 2767927687

29. Puntervoll, P., Ruud, M., Bruseth, L. J., Kleivdal, H., Høgh, B. T., Benz, R. \& Jensen, H. B. (2002). Structural characterization of the fusobacterial nonspecific porin FomA suggests a 14-stranded topology, unlike the classical porins. Microbiology, 148, 33953403.

30. Pocanschi, C. L., Apell, H.-J., Puntervoll, P., Høgh, B. T., Jensen, H. B., Welte, W. \& Kleinschmidt, J. (2006). The major outer membrane protein of Fusobacterium nucleatum (FomA) folds and inserts into 
lipid bilayers via parallel folding pathways. J. Mol. Biol. 355, 548-561.

31. Colombini, M. (2004). VDAC: the channel at the interface between mitochondria and the cytosol. Mol. Cell. Biochem. 256-257, 107-115.

32. Kleinschmidt, J. H. \& Tamm, L. K. (1999). Timeresolved distance determination by tryptophan fluorescence quenching: probing intermediates in membrane protein folding. Biochemistry, 38, 4996-5005.

33. Bitto, E. \& McKay, D. B. (2004). Binding of phagedisplay-selected peptides to the periplasmic chaperone protein SurA mimics binding of unfolded outer membrane proteins. FEBS Letters, 568, 94-98.

34. Kim, S., Malinverni, J. C., Sliz, P., Silhavy, T. J., Harrison, S. C. \& Kahne, D. (2007). Structure and function of an essential component of the outer membrane protein assembly machine. Science, 317, 961-964.

35. Robert, V., Volokhina, E. B., Senf, F., Bos, M. P., Van Gelder, P. \& Tommassen, J. (2006). Assembly factor Omp85 recognizes its outer membrane protein substrates by a species-specific C-terminal motif. PLoS Biol. 4, e377.

36. Rizzitello, A. E., Harper, J. R. \& Silhavy, T. J. (2001). Genetic evidence for parallel pathways of chaperone activity in the periplasm of Escherichia coli. J. Bacteriol. 183, 6794-6800.

37. Johanson, K. O., Wetlaufer, D. B., Reed, R. G. \& Peters, T., Jr (1981). Refolding of bovine serum albumin and its proteolytic fragments. Regain of disulfide bonds, secondary structure, and ligand-binding ability. J. Biol. Chem. 256, 445-450.

38. Shanmugavadivu, B., Apell, H. J., Meins, T., Zeth, K. \& Kleinschmidt, J. H. (2007). Correct folding of the $\beta$-barrel of the human membrane protein VDAC requires a lipid bilayer. J. Mol. Biol. 368, 66-78.

39. Kleinschmidt, J. H. \& Tamm, L. K. (2002). Secondary and tertiary structure formation of the $\beta$-barrel membrane protein OmpA is synchronized and depends on membrane thickness. J. Mol. Biol. 324, 319-330.

40. Ramakrishnan, M., Pocanschi, C. L., Kleinschmidt, J. H. \& Marsh, D. (2004). Association of spin-labeled lipids with $\beta$-barrel proteins from the outer membrane of Escherichia coli. Biochemistry, 43, 11630-11636.

41. Ferguson, A. D., Welte, W., Hofmann, E., Lindner, B., Holst, O., Coulton, J. W. \& Diederichs, K. (2000). A conserved structural motif for lipopolysaccharide recognition by procaryotic and eucaryotic proteins. Structure, 8, 585-592.

42. Ramakrishnan, M., Qu, J., Pocanschi, C. L., Kleinschmidt, J. H. \& Marsh, D. (2005). Orientation of $\beta$-barrel proteins OmpA and FhuA in lipid membranes. chain length dependence from infrared dichroism. Biochemistry, 44, 3515-3523.

43. Vinogradov, E. V., Van Der Drift, K., Thomas-Oates, J. E., Meshkov, S., Brade, H. \& Holst, O. (1999). The structures of the carbohydrate backbones of the lipopolysaccharides from Escherichia coli rough mutants F470 (R1 core type) and F576 (R2 core type). Eur. J. Biochem. 261, 629-639.

44. Müller-Loennies, S., Holst, O. \& Brade, H. (1994). Chemical structure of the core region of Escherichia coli J-5 lipopolysaccharide. Eur. J. Biochem. 224, 751-760.

45. Ladokhin, A. S., Wang, L., Steggles, A. W. \& Holloway, P. W. (1991). Fluorescence study of a mutant cytochrome b5 with a single tryptophan in the membranebinding domain. Biochemistry, 30, 10200-10206.

46. van Holde, K. E., Curtis Johnson, W. \& Shing Ho, P. (2006). Principles of Physical Biochemistry, 2 edit. Pearson Prentice Hall, London.

47. Cantor, C. R. \& Schimmel, P. (1980). Biophysical Chemistry. Part II: Techniques for the Study of Biological Structure and Function. W. H. Freeman and Company, New York.

48. Lowry, O. H., Rosebrough, N. J., Farr, A. L. \& Randall, R. J. (1951). Protein measurement with the Folin phenol reagent. J. Biol. Chem. 193, 265-275.

49. Gasteiger, E., Hoogland, C., Gattiker, A., Duvaud, S., Wilkins, M. R., Appel, R. D. \& Bairoch, A. (2005). Protein identification and analysis tools on the ExPASy server. In The Proteomics Protocols Handbook), Walker, J. M., ed), pp. 571-607, Humana Press, Totawa. 